\title{
Effect of Reservoir Heterogeneity on the Vertical Migration of Hydraulic Fractures
}

Hisanao Ouchi, John T. Foster, Mukul M. Sharma, the University of Texas at Austin

\section{Abstract}

The effect of different types of vertical reservoir heterogeneities on fracture propagation was systematically investigated. A fully 3-D, poroelastic model that does not prescribe the crack propagation path is used to estimate the fracture geometry in vertically heterogeneous rocks. Complex fracture trajectories are shown to occur and this limits fracture height growth. It is shown that the presence of bedding planes, layer interfaces and even smaller scale heterogeneities can lead to fracture turning, kinking or branching. The mechanisms that control these characteristic fracture propagation behaviors ("turning", "kinking", and "branching") near the layer interface are explored in detail. In layered systems, the mechanical property contrast between layers, the dip angle, the stress contrast and the mechanical properties of the layer interface all play an important role in controlling the fracture trajectory. Conditions under which each type of behavior is expected to occur are clearly delineated.

\section{Introduction}

Shale gas/oil reservoirs inherently contain heterogeneities at multiple length scales (Filder, 2011; Mokhtari et al., 2014). Examples of this include micrometer scale heterogeneities at the grain scale, and meter scale heterogeneities such as bedding planes (Maxwell et al., 2009; Walton and McLennan, 2013). All of them may influence hydraulic fracture propagation. However, the effect of these heterogeneities on fracture propagation has not been fully investigated. The effect of layer-scale heterogeneity on fracture propagation was investigated by several authors (Gu and Siebrits, 2008; Thiercelin et al., 1989; van Eekelen, 1982). They studied the effect of such layering on width growth in planar fractures. However, contrasts in mechanical properties and weak interfaces between different layers, not only affect the width of planar fractures, but can also cause complicated fracture propagation patterns. As Fisher et al. (Fisher and Warpinski, 2012) point out, fractures can show complicated behavior such as "bending", "kinking", and "offsetting" at layer interfaces. When these phenomena occur, fracture height growth is limited when compared with a planar fracture due to the narrower fracture width at the fracture turning point. Moreover, this is likely to cause proppant bridging or screen out (Fisher and Warpinski, 2012). Therefore, understanding the mechanism of fracture propagation near layer interfaces is important from a fracture design viewpoint. A few authors have tried to investigate such complicated fracture propagation behavior near layer interfaces. Based on an energy analysis and comparisons with experimental results, Wu et al. (Wu et al., 2004) have shown that hydraulic fractures can turn (bend), kink, and be arrested at the layer boundary when fractures go from a softer layer to a harder layer. Zhang et al. (Zhang et al., 2007) have demonstrated that the magnitude of far field stresses acting on a layer interface and the frictional strength of a layer interface mainly affect fracture turning along the layer interface. Garcia et al. (Garcia et al., 2013) have shown that the contrast in fracture toughness could also cause different types of complicated fracture propagation behavior ("turning", 
"kinking", and "offsetting") near the layer interface. However, a comprehensive study of the conditions under which the different fracture propagation behavior should be expected to occur for multi-layered rocks has not been conducted yet. Part of the reason for this has been the inability of past models to simulate complex fracture propagation patterns without prescribing the fracture propagation direction. Allowing the fracture to propagate in any direction (controlled by the underlying physics and material properties) and to branch into multiple fractures is essential to simulating fracture complexity in heterogeneous rocks.

Peridynamics is a recently developed nonlocal theory of continuum mechanics that accounts for discontinuities such as fractures naturally (Silling and Askari, 2005; Silling et al., 2007; Silling et al., 2010b). Its integral formulation eliminates the spatial derivatives in the classical theory that are incompatible with the nature of cracks. The validity and applicability of peridynamics to solid and fracture mechanics has been demonstrated (Askari et al., 2008; Askari et al., 2006; Bobaru, 2007; Silling and Bobaru, 2005; Silling et al., 2010a; Xu et al., 2007). We have recently derived a new peridynamics fluid flow formulation (Katiyar et al., 2014) and developed a new peridynamics-based hydraulic fracturing model (Ouchi et al., 2015a; Ouchi et al., 2015b). This model can simulate non-planar, multiple fracture growth in arbitrarily heterogeneous reservoirs by solving fracture propagation, deformation, fracturing fluid pressure, and pore pressure simultaneously.

In order to investigate the effect of vertical heterogeneity on fracture propagation at different scales, we use this new peridynamics-based hydraulic fracturing model to simulate fracture propagation in vertically heterogeneous porous media. We systematically investigate how the four types of phenomena ("crossing", "turning (bending)" "kinking", and "branching") take place near the layer interface depending on the contrast in the layer mechanical properties, the layer dip angle and the properties of the interface.

\section{Mathematical Model}

The details of the model used in this study have been presented by Ouchi et. al (Ouchi et al., 2015a; Ouchi et al., 2015b). This model takes into account fracture propagation and poroelastic effects by simultaneously solving the three governing equations for mechanics, porous flow, and fracture flow. In the following section, we briefly explain the basic concepts of peridynamics, the governing equations and the constitutive relations for mechanics and for porous flow in the matrix and the fracture.

\subsection{Basic Concepts of Peridynamics (Horizon, Bond, State, and Reference Configuration)}

Peridynamics theory is the non-local continuum formulation of the classical solid mechanics theory. In peridynamics, as shown in Fig. 1, a material is assumed to be composed of material points of known mass and volume (element) and every element interacts with all the neighboring elements inside a nonlocal region, referred as "horizon", around it. In the state-based peridynamic formulation, mathematical objects called peridynamic states have been introduced for convenience (Silling et al., 2007). Peridynamic states depend upon position and time, shown in square brackets, and operate on a vector, shown in angled brackets, connecting any two material 
points. Depending on whether the value of this operation is a scalar or vector, the state is called a scalar-state or a vector-state respectively. In this paper, to differentiate the two states, the vector-states are expressed with the underlined and bold character and the scalar-states are denoted with the underlined character.

As shown in Fig. 2, the reference position of elements $\mathbf{x}$ and $\mathbf{X}^{\prime}$ in the reference configuration is given by the reference position vector-state $\underline{\mathbf{X}}$ in state-based peridynamics theory. On the other hand, the relative position of the same material points in the deformed configuration is given by the deformed position vector-state $\underline{\mathbf{Y}}$.

$$
\begin{aligned}
& \underline{\mathbf{X}}\langle\xi\rangle=\mathbf{x}^{\prime}-\mathbf{x}=\xi \\
& \underline{\mathbf{Y}}\langle\xi\rangle=\mathbf{y}\left(\mathbf{x}^{\prime}\right)-\mathbf{y}(\mathbf{x})=\xi+\boldsymbol{\eta}
\end{aligned}
$$

Where, $\mathbf{y}$ is a deformed position vector (unit: [m]), $\xi$ is a bond vector (unit: [m]), and $\boldsymbol{\eta}$ relative displacement vector (unit: $[\mathrm{m}]$ ).

\subsection{Governing Equation and Constitutive Relation for Mechanics}

The generalized state based peridynamics equation of motion (conservation of linear momentum) is given as an integral functional summing up the force densities in all the connected bonds over the horizon.

$$
\begin{aligned}
\rho \ddot{\mathbf{u}} & =\int_{H_{x}}\left(\underline{\mathbf{T}}[\mathbf{x}]\langle\xi\rangle-\underline{\mathbf{T}}\left[\mathbf{x}^{\prime}\right]\langle-\xi\rangle\right) d V_{\mathbf{x}^{\prime}}+\mathbf{b}[\mathbf{x}] \\
& =\int_{H_{x}}\left(\underline{t}[\mathbf{x}, t]\langle\xi\rangle \frac{\xi+\boldsymbol{\eta}}{\|\xi+\boldsymbol{\eta}\|}-\underline{t}\left[\mathbf{x}^{\prime}, t\right]\langle-\xi\rangle \frac{-(\xi+\boldsymbol{\eta})}{\|\xi+\boldsymbol{\eta}\|}\right) d V_{\mathbf{x}^{\prime}}+\mathbf{b}[\mathbf{x}] \\
& =\int_{H_{x}}\left(\underline{t}[\mathbf{x}, t]\langle\xi\rangle+\underline{t}\left[\mathbf{x}^{\prime}, t\right]\langle-\xi\rangle\right) \frac{\xi+\boldsymbol{\eta}}{\|\xi+\boldsymbol{\eta}\|} d V_{\mathbf{x}^{\prime}}+\mathbf{b}[\mathbf{x}]
\end{aligned}
$$

Where, $\mathbf{b}$ is an external force density (unit $\left[\mathrm{N} / \mathrm{m}^{3}\right]$ ) $\underline{t}$ is peridynamic force scalar-state (unit: $\left[\mathrm{N} / \mathrm{m}^{6}\right]$ ), $\underline{\mathbf{T}}$ is peridynamic force vector-state (unit: $\left[\mathrm{N} / \mathrm{m}^{6}\right]$ ), $\ddot{\mathbf{u}}$ is acceralation vector (unit: $\left[\mathrm{m} / \mathrm{s}^{2}\right]$ ), $V$ is material volume (unit: $\left[\mathrm{m}^{3}\right]$ ), and $\rho$ is material density (unit: $\left[\mathrm{kg} / \mathrm{m}^{3}\right]$ ).

To consider the effect of pore pressure (poroelastic effect) on displacement, we introduce the concept of the effective force scalar-state (Turner, 2012) in our model. The constitutive relation of effective force scalar-state $\underline{t}[\mathbf{x}, t]\langle\xi\rangle$ in the poroelastic material is given as follows,

$$
\begin{aligned}
\underline{t}[\mathbf{x}, t]\langle\xi\rangle & =\frac{3 \Gamma_{1}(K \theta-\alpha P)}{m} \underline{\omega} \underline{x}\langle\xi\rangle+\frac{15 \Gamma_{2} G}{m} \underline{\omega}\left(e-\frac{\theta}{3} \underline{x}\langle\xi\rangle\right) \\
& =\frac{\left\{\left(3 \Gamma_{1} K-5 \Gamma_{2} G\right) \theta-3 \Gamma_{1} \alpha P\right\} \underline{\omega}\|\xi\|}{m}+\frac{15 \Gamma_{2} G}{m} \underline{\omega}(\|\xi+\boldsymbol{\eta}\|-\|\xi\|)
\end{aligned}
$$




$$
\begin{aligned}
& m=\underline{\omega} \underline{x}\langle\xi\rangle \bullet \underline{x}\langle\xi\rangle=\int_{H_{x}} \underline{\omega} \underline{x}\langle\xi\rangle \underline{x}\langle\xi\rangle d A_{\mathbf{x}^{\prime}} \\
& \theta=\frac{3}{m} \underline{\omega} \underline{x} \bullet \underline{e}=\frac{3}{m} \int_{H_{x}} \underline{\omega} \underline{x}\langle\xi\rangle e\langle\xi\rangle d V_{\mathbf{x}^{\prime}}
\end{aligned}
$$

Where, $G$ is shear modulus (unit: $[\mathrm{Pa}]$ ), $K$ is bulk modulus (unit $[\mathrm{Pa}]$ ), $m$ is weighted volume (unit $\left[\mathrm{m}^{3}\right]$ ), $P$ is pore pressure (unit: $[\mathrm{Pa}]$ ), $\theta$ is dilatation (unit: dimensionless), $\underline{\omega}$ is influence function, $\Gamma_{1}$ is scaling factor for dimension (3-D: 1.0, 2-D: 2/3), $\Gamma_{2}$ is scaling factor2 for dimension (3-D: 1.0, 2-D, 8/15). Note that if the two elements have the different mechanical properties, based on the Equation (4), the force interaction between the two elements (element $\mathrm{i}$ and element $\mathrm{j}$ ) takes the following form (kind of arithmetic averaging formulation) in the original peridynamics theory.

$$
\left.t[\mathbf{x}, t]\langle\xi\rangle+t\left[\mathbf{x}^{\prime}, t\right]\langle-\xi\rangle=\frac{\underline{\omega}}{m}\left[\begin{array}{l}
\left(3 \Gamma_{1} K_{i}-5 \Gamma_{2} G_{i}\right) \theta_{i} \\
+\left(3 \Gamma_{1} K_{j}-5 \Gamma_{2} G_{j}\right) \theta_{j} \\
-3 \Gamma_{1} \alpha\left(P_{i}+P_{j}\right) \\
+15 \Gamma_{2}\left(G_{i}+G_{j}\right) e_{i j}
\end{array}\right\}\left\|\mathbf{x}_{j}-\mathbf{x}_{i}\right\|\right]
$$

However, according to the result of the numerical experiments (Ouchi, 2016), Equation (7) shows undesirable smoothing of displacements near the layer interface if the contrast of a mechanical property is high. Therefore, we apply the following formulation (harmonic averaging modulus) instead of the original one in this research. However, more work is necessary for proving the validity of this constitutive relation.

$$
\left.t[\mathbf{x}, t]\langle\xi\rangle+t\left[\mathbf{x}^{\prime}, t\right]\langle-\xi\rangle=\frac{\underline{\omega}}{m}\left[\begin{array}{l}
\int\left(3 \Gamma_{1} \bar{K}-5 \Gamma_{2} \bar{G}\right)\left(\theta_{i}+\theta_{j}\right) \\
-3 \Gamma_{1} \alpha\left(P_{i}+P_{j}\right) \\
+15 \Gamma_{2} \bar{G} e_{i j}
\end{array}\right\}\left\|\mathbf{x}_{j}-\mathbf{x}_{i}\right\|\right]
$$

Material failure (nucleation of fracture) can be expressed through a damage model in peridynamics. If the energy stored in the bond $\left(=\omega_{\xi}\right)$ exceeds the critical energy $\left(=\omega_{c}\right)$ which is related to the energy release rate, the mechanical force interaction between the two elements (bond) disappears (Foster et al., 2011). This bond failure is numerically implemented by multiplying the following boolean function to the mechanical term in force scalar-state.

$$
\beta\langle\xi\rangle=\left\{\begin{array}{l}
0\left(\omega_{\xi}>\omega_{c}\right) \\
1 \text { (otherwise) }
\end{array}\right.
$$

Once a bond is broken, since all the mechanical terms disappears due to the Boolean function $\beta$, the force scalar-state is given as follows, 


$$
\underline{t}[\mathbf{x}, t]\langle\xi\rangle=-\frac{3 \Gamma_{1} \alpha P \underline{\omega}\|\xi\|}{m}
$$

Based on the ratio of broken bond, Damage $d$ is defined as follows,

$$
d(\mathbf{x})=1-\frac{\int_{H_{\mathbf{x}}} \beta\langle\xi\rangle d V_{\mathbf{x}^{\prime}}}{\int_{H_{\mathbf{x}}} d V_{\mathbf{x}^{\prime}}}
$$

Where, $d$ is damage. Damage at any point and time varies from 0 to 1 , with 1 representing all the bonds attached to a point broken.

In our model, it is regarded that fracture space is created when the following two conditions are satisfied simultaneously. This condition is referred to as "dual points".

- Damage exceeds the critical damage at both the two nearest elements

- The energy density in the bond between the two nearest elements exceeds the critical energy density (in other words, the bond between the two nearest elements breaks).

Once a fracture space is created, to apply a non-local traction boundary condition $\left(=P_{f}\right)$ to the fracture surface, the force scalar-state is replaced from Equation (10) to Equation (12) in all the broken bonds passing through the fracture surface (Ouchi et al., 2015b).

$$
\underline{t}[\mathbf{x}, t]\langle\xi\rangle=-\frac{3 \Gamma_{1} P_{f} \underline{\omega}\|\xi\|}{m}
$$

\subsection{Governing Equation and Constitutive Relation for Porous Flow}

The governing equation for porous flow (mass conservation equation of porous fluid) is given by the following formulation (Katiyar et al., 2014).

$$
\frac{\partial}{\partial t}\left(\rho_{w}[\mathbf{x}] \phi[\mathbf{x}]\right)=\int_{H_{x}}\left(\underline{Q}[\mathbf{x}, t]\langle\xi\rangle-\underline{Q}\left[\mathbf{x}^{\prime}, t\right]\langle-\xi\rangle\right) d V_{\mathbf{x}^{\prime}}+I[\mathbf{x}]
$$

Where, $I[\mathbf{x}]$ is the source term for leak-off, $\underline{Q}[\mathbf{x}, t]\langle\xi\rangle$ is flow scalar-state, $\phi$ is porosity, and $\rho_{w}$ is porous fluid density (unit: $\left[\mathrm{kg} / \mathrm{m}^{3}\right]$ ).

The constitutive relation in flow scalar-state $\underline{Q}[\mathbf{x}, t]\langle\xi\rangle$ is given as follows (Gunzburger and Lehoucq, 2010; Seleson, 2010), 


$$
\underline{Q}[\mathbf{x}, t]\langle\xi\rangle=\frac{\gamma}{2 \mu} \underline{\omega}\langle\xi\rangle \rho_{0} \frac{\xi\left(\mathbf{k}[\mathbf{x}]-\frac{1}{2+d_{\text {num }}} \operatorname{tr}(\mathbf{k}[\mathbf{x}]) \mathbf{I}\right) \xi}{\|\xi\|^{4}}\left(P\left[\mathbf{x}^{\prime}\right]-P[\mathbf{x}]\right)
$$

Where, $d_{\text {num }}$ is dimension (2-D: 2, 3-D: 3), $\mathbf{k}$ is permeability tensor, $\gamma$ is a scaling factor depending on the horizon size, the dimension, and influence function (if $\underline{\omega}=1$ (default value for fluid flow calculation), 2-D: $\gamma=8 / \pi \delta^{2}, 3-\mathrm{D}: \gamma=45 / 4 \pi \delta^{3}$ ), $\mu$ is viscosity (unit: [Pa s]).

To take into account the effect of the deformation of porous body on porous flow, the porosity in Equation (13) is evaluated by the following formulation derived by Tran et. al (Tran et al., 2004).

$$
\phi^{(n+1)}[\mathbf{x}]=\phi^{(n)}[\mathbf{x}]\left(1-C_{r} \Delta P\right)+\alpha\left(1-\varepsilon_{v}^{(n)}\right)\left\{C_{r} \Delta P+\theta_{\text {local }}^{(n+1)}-\theta_{\text {local }}^{(n)}\right\}
$$

Where, $C_{r}$ is compressibility of rock grain $\left(=1 / K_{m}\right.$, unit: $\left.[1 / \mathrm{Pa}]\right), K_{m}$ is bulk modulus of rock grain (unit: $[\mathrm{Pa}])$, upscript ${ }^{(n)}$ represents time step (n), $\alpha$ is Biot's coefficient $\left(=1-K / K_{m}\right), \varepsilon_{v}$ is volumetric strain, and $\theta_{\text {local }}$ is the local dilatation (dilatation in the limited horizon size $(<=1.5$ element size $)$ ).

\subsection{Governing Equation and Constitutive Relation for Fracture Flow}

The governing equation for fracture flow is given as follows,

$$
\frac{\partial}{\partial t}\left(\rho_{f}[\mathbf{x}] \phi_{f}[\mathbf{x}]\right)=\int_{H}\left(\underline{Q}_{f}[\mathbf{x}, t]\langle\xi\rangle-\underline{Q}_{f}\left[\mathbf{x}^{\prime}, t\right]\langle-\xi\rangle\right) d V_{\mathbf{x}^{\prime}}+R_{f}[\mathbf{x}]-I[\mathbf{x}]
$$

Where, $\underline{Q}_{f}[\mathbf{x}, t]\langle\xi\rangle$ is fracture flow scalar-state, $R_{f}$ is the source term for injection (unit: $\left[\mathrm{kg} /\left(\mathrm{m}^{3} \mathrm{~s}\right)\right], \phi_{f}$ is the ratio of fracture space to the element volume, and $\rho_{f}$ is density of fracturing fluid (unit: $\left[\mathrm{kg} / \mathrm{m}^{3}\right]$ ).

Through lubrication theory, fracture flow inside the dual point is expressed as a flow formulation with fracture permeability $k_{f}$ which is the function of a fracture width $w$.

$$
\underline{Q}_{f}[\mathbf{x}, t]\langle\xi\rangle=\frac{\gamma}{2} \frac{\rho_{f}[\mathbf{x}]}{\mu_{f}} \frac{\Gamma_{3} k_{f}[\mathbf{x}]}{\|\xi\|^{2}}\left(P_{f}\left[\mathbf{x}^{\prime}\right]-P_{f}[\mathbf{x}]\right)
$$

Where, $k_{f}$ is fracture permeability (unit: $\left.\left[\mathrm{m}^{2}\right]\right), \Gamma_{3}$ is correction factor $\left(=\frac{2}{2+d_{\text {num }}} \frac{w}{l_{e l m}}, l_{\text {elm }}:\right.$ element length), and $\mu_{f}$ is fracturing fluid viscosity (unit: [Pa s]). 
For an intact rock, the present model solves only Equations (3) and (13) (only displacements and pore pressure are solved in each element). However, once the condition of "dual point" is satisfied, an element is assumed to represent the fracture space as well and Equation (16) is additionally solved (fracturing fluid pressure is additionally solved in the elements where fracture space appears). Hence, the number of primary unknowns keeps on increasing with time in this model.

Fracture porosity at the elements where fracture space is included is defined as follows,

$$
\phi_{f}= \begin{cases}0 & \text { for } d \leq d_{c r} \\ \theta_{\text {local }}-\theta_{\text {local } @ \omega_{c}} & \text { for } d>d_{c r}\end{cases}
$$

Where, $\theta_{\text {local } @ \omega_{c}}$ is local dilatation where stretch of the bonds attached to the dual point is restricted to the critical stretch beyond which a bond breaks.

Fracture permeability is expressed as a function of width through lubrication theory. Fracture width $w$ is approximated based on a fracture porosity $\phi_{f}\left(w=l_{\text {elm }} \phi_{f}\right)$.

$$
k_{f}=\frac{w^{2}}{12}
$$

Leak-off is modeled through a source term $I[\mathbf{x}]$ as follows,

$$
\begin{aligned}
& I[\mathbf{x}]=-\frac{\rho_{f}[\mathbf{x}] k_{\text {leak }} A}{V \mu_{f}} \frac{d P}{d l}=\frac{\rho_{f}[\mathbf{x}] k_{\text {leak }}\left(P_{f}[\mathbf{x}]-P[\mathbf{x}]\right)}{l_{\text {elm }} \mu_{f}\left(l_{\text {elm }} / 2\right)} \\
& k_{\text {leak }}=\mathbf{n}_{\text {surf }} \cdot\left(\mathbf{k}[\mathbf{x}] \mathbf{n}_{\text {surf }}\right)
\end{aligned}
$$

Where, $n_{\text {surf }}$ is a unit normal vector perpendicular to a fracture surface.

\subsection{Initial and Boundary Conditions}

If the simulation domain is composed of multiple materials, such as layers which have different Young's modulus and Poisson's ratio, it is difficult to represent the in-situ stress state by just applying a traction boundary condition due to stress concentration along the different material boundaries. In order to avoid stress concentration between the two different materials and to impose arbitrary initial stress condition directly on material points, we introduce Silling's definition (Silling et al., 2007) of the peridynamic background force vector-state $T_{0}[\mathbf{x}]\langle\xi\rangle$ which allows us to allocate effective Piola-Kirchhoff stress tensor $\boldsymbol{\sigma}_{\text {eff }}$ in the classical theory. The peridynamic 
background force vector-state for the material point $\mathbf{x}$ with initial stress condition $\boldsymbol{\sigma}_{e f f}$ is given by the following formulation.

$$
\begin{gathered}
\underline{T}_{0}[\mathbf{x}]\langle\xi\rangle=\underline{\omega}_{e f f} \mathbf{K}^{-1} \xi \\
\mathbf{K}=\int_{H_{\mathbf{x}}} \underline{\omega} \xi \otimes \xi d V_{\mathbf{x}^{\prime}}
\end{gathered}
$$

Where, $\mathbf{K}$ is non-local shape tensor (unit: $\left[\mathrm{m}^{2}\right]$ ), $\underline{T}_{0}$ is background force vector-state (unit: $\left[\mathrm{N} / \mathrm{m}^{6}\right]$ ), and $\otimes$ is dyadic product. By adding Equation (22) to the ordinary peridynamic force vector-state (2.8) as a background force vector-state, an arbitrary initial stress condition can be allocated in the simulation domain.

$$
\underline{T}[\mathbf{x}, t]\langle\xi\rangle=\underline{t}[\mathbf{x}, t]\langle\xi\rangle \frac{\xi+\boldsymbol{\eta}}{\|\xi+\boldsymbol{\eta}\|}+T_{0}[\mathbf{x}]\langle\xi\rangle
$$

Since we assume force equilibrium as the initial condition, the following force density is applied as a traction boundary condition in the case where the background force vector-state is given (under the force equilibrium condition, the body force given from the boundary must be equivalent to the negative value of the summation of the back ground force vector-state inside the model domain.)

$$
b[\mathbf{x}]=\int_{B_{\text {outside }}}\left\{T_{0}[\mathbf{x}]\langle\xi\rangle-T_{0}\left[\mathbf{x}^{\prime}\right]\langle-\xi\rangle\right\} d V_{\mathbf{x}^{\prime}}=-\int_{B_{\text {inside }}}\left\{T_{0}[\mathbf{x}]\langle\xi\rangle-T_{0}\left[\mathbf{x}^{\prime}\right]\langle-\xi\rangle\right\} d V_{\mathbf{x}^{\prime}}
$$

\section{Results and Discussion}

\subsection{Investigation of Fracture Propagation Behavior near a Layer Boundary}

\subsubsection{Model Description}

To investigate the effect of mechanical property contrast and layer dip angle on fracture propagation near a layer boundary, as shown in Fig. 3, we constructed a two layer model and conducted a comprehensive parametric study of the model. The model domain $(30 \mathrm{~cm} \times 15 \mathrm{~cm})$ is divided into $150 \times 75$ elements. The upper two-thirds of the domain (10 cm from the top) is assigned to Layer 1 and the lower one-third of the domain $(5 \mathrm{~cm}$ from the bottom) is assigned to Layer 2. For fluid flow calculations, a no-flow boundary condition is applied to every boundary (top, bottom, left side, and right side). For mechanical calculations, a normal stress of magnitude $\sigma_{V}$ is applied as a maximum principal stress at the top boundary, and a normal stresses of magnitude $\sigma_{H 1}$ (horizontal stress for layer 1) and $\sigma_{H_{2}}$ (horizontal stress for layer 2) are applied to Layer 1 and Layer 2 respectively. Note that to avoid stress concentration near the layer interface due to the different magnitude of strains in the two different layers, the same stress condition as the far field stresses mentioned above are directly assigned to each element as a background force vector (in the same manner as explained in the above section). As shown in Fig. 3 , to simulate fracture propagation, a fluid injection point is set as "dual injection point" at the bottom of the model 
and no vertical displacement is allowed at the bottom boundary so that the boundary can only deform in the horizontal direction. By changing the mechanical properties, horizontal stress, and layer dip angle, we investigated the fracture propagation behavior near the layer interface. The parameters we changed in this section are summarized in Table 1. Calculation settings are summarized in Table 2.

\subsubsection{Effect of Young's Modulus, Fracture Toughness, Horizontal/Vertical Stress Contrast, and Layer Dipping Angle}

The effect of Young's modulus, fracture toughness contrast, horizontal/vertical stress difference, and layer dipping angle are summarized in Fig. 4, Fig. 5, and Fig. 6. All other properties are fixed as shown in Table 3. Different fracture propagation patterns are observed by changing the stress contrast $\left(=\sigma_{V}-\sigma_{H}\right.$ plotted on the $\mathrm{x}$ axis), fracture toughness of the first layer (plotted on the y axis), and layer dip angle. As shown in Fig. 4 - Fig. 6 , three types of fracture propagation behavior near the layer interface are observed: "turning", "branching", and "kinking". For each fracture propagation behavior, we first explain the propagation mechanism based the results of some specific cases. After that, we discuss the effect of each parameter on the propagation behavior.

\subsubsection{Fracture Turning}

\section{(1) Mechanism}

Fig. 7 shows the bottom-hole (BHP) change with time in the two different cases where only the fracture toughness contrast is different $\left(1.41 \mathrm{MPa} \mathrm{m}^{0.5}\right.$ and $\left.2.0 \mathrm{MPa} \mathrm{m}^{0.5}\right)$. All the other parameters are fixed as follows: layer dip angle $=0$ degree, Young's modulus contrast $1.0(=10 \mathrm{GPa} / 10 \mathrm{GPa})$, and principal stress difference $=1$ MPa. As shown in Fig. 7, the fracture passes through the layer interface in the case where fracture toughness of the first layer $\left(\mathrm{K}_{\mathrm{IC} 1}\right)$ is $1.41 \mathrm{MPa} \mathrm{m}^{0.5}$ (we call this case the "crossing" case) while the fracture turns along the layer interface in the case where the fracture toughness in Layer 1 is $2.0 \mathrm{MPa} \mathrm{m}^{0.5}$ (we call this case the "turning" case).

In both cases, at the early stage $(\mathrm{t}<0.27 \mathrm{sec})$, the BHP decreases with time due to the fracture volume expansion. Then, from $(0.27 \mathrm{sec}<=\mathrm{t}<0.5 \mathrm{sec})$, the BHP begins to increase since the fracture propagation stops at the layer interface due to the higher energy requirement for breaking bonds in the first layer compared to the second layer. During this time, the fracture width continues to increase due to the BHP increase. As shown in Fig. 8, the fracture width expansion causes the bonds to stretch both in the horizontal direction in Layer 1 and in the bonds across the layer interface between Layer 1 and Layer2. Therefore, if the horizontal bonds in Layer1 shown in Fig. 8 break sooner than the bonds across the layer interface, the fracture crosses the layer interface. Conversely, if all the bonds across the layer interface break sooner than the horizontal bonds in Layer1 due to a shear like displacement along the layer interface, the fracture turns along the layer interface. In essence, if the bonds' connection across the layer interface break faster than the bonds in the upper layer, "turning" occurs. Please note that if "turning" does not occur, "crossing" always occurs in our models since the models investigated in this research are two dimensional. However, in the real (three dimensional) situations, no "turning" does not always mean "crossing". Fracture can stop at a layer interface and can propagate in the $3^{\text {rd }}$ lateral direction (maximum horizontal stress direction). Part of "crossing" results in this research represent fracture "stopping" at the layer interface in the real 
situations. In the next section, we discuss how the following parameters (fracture toughness contrast, principal stress difference, Young's modulus contrast, and layer dip angle) affect the timing of bond failure.

\section{(2) Effect of fracture toughness and principal stress difference on turning}

As shown in Fig. 4, the turning criterion is mainly affected by the principal stress difference between vertical and horizontal stresses, and the toughness contrast between the two layers. A hydraulic fracture is more likely to turn along the layer interface for a lower principal stress difference and a higher fracture toughness contrast. A two to four times fracture toughness contrast $\left(K_{I C 1} / K_{I C 2}>2-4\right)$ is necessary for the fracture to turn under a low principal stress difference condition $(\Delta \sigma<1.0 \mathrm{MPa}$ ), while a fracture toughness contrast of about eight to ten times is necessary for a high principal stress difference condition ( $\triangle \sigma \square 20 M P a$ ). Since the published values of fracture toughness range from $0.2 \approx 3.0 \mathrm{MPa} \sqrt{\mathrm{m}}$ (Rijken and Cooke, 2001; Senseny and Pfeifle, 1984; Thiercelin et al., 1989), the possible fracture toughness contrast is less than fifteen even in extreme cases. In many cases, the contrast is less than around 2.0 to 3.0 at best. This suggests that fracture turning along a layer interface is unlikely to occur especially in deep reservoirs. This is consistent with field observations by tiltmeter surveys. Horizontal fracture propagation is not commonly observed deeper than $4000 \mathrm{ft}$, whereas a horizontal fracture component begins to appear in measurements made for fractures shallower than $4000 \mathrm{ft}$, as reported by Fisher et al. (Fisher and Warpinski, 2012). In our model the tendencies related to toughness contrast and principal stress difference are explained by using the formulation for the critical displacement $\left\|\boldsymbol{\eta}_{\text {crit }}\right\|$ (the bond displacement just before the bond breaks). If we assume that the influence function is given as $\underline{\omega}=1 / r$ (default of the simulator, $0<r \leq \delta$ ), the far field effective stress tensor is given as $\boldsymbol{\sigma}_{e f f}=\left(\begin{array}{cc}\sigma_{H} & 0 \\ 0 & \sigma_{V}\end{array}\right)$, Poisson's ratio $=0.25$, Biot's coefficient $\alpha=0.0$ (for simplicity), weighted volume $m$ and shape tensor $\mathbf{K}$ are given by the following analytical forms respectively in Equation (24), the critical displacement $\left\|\boldsymbol{\eta}_{\text {crit }}\right\|$ in the horizontal and vertical direction across the layer interface can be analytically estimated as follows (Ouchi, 2016). Note that, in Equation (27), we assume that the critical energy density for the bond across the layer interface is given as the smaller value of the critical energy densities of the two layers (we assume that the critical energy density for Layer 2 is smaller than Layer 1 in this case).

$$
\begin{gathered}
\|\boldsymbol{\eta}\|_{\text {crit_hori }}=\frac{5 \sqrt{r}}{8 E_{1}}\left(\sqrt{\frac{9 \pi}{8}} K_{I C 1}+\sqrt{r} \sigma_{H}\right) \quad(0<r \leq \delta) \\
\|\boldsymbol{\eta}\|_{\text {crit_vert }}=\frac{5 \sqrt{r}}{8 \bar{E}}\left(\sqrt{\frac{9 \pi \bar{E}}{8 E_{2}}} K_{I C 2}+\sqrt{r} \sigma_{V}\right) \quad(0<r \leq \delta)
\end{gathered}
$$

Where, $\bar{E}$ is the average Young's modulus between Layer 1 and Layer 2 (unit: [Pa]). Equation (26) and (27) clearly shows that a higher fracture toughness and a higher initial stress require a higher displacement to break a 
bond. Therefore, as shown in Equation (26), if the fracture toughness in Layer 1 is high, fracture propagation in Layer 1 is difficult and it is difficult to cross the layer interface. In addition, as shown in Equation (27), if the initial vertical stress is high, it is difficult to break the bonds across the layer interface (difficult to turn along the layer interface).

\section{(3) Effect of Young's modulus on Turning}

As shown in Fig. 4, the effect of Young's modulus contrast on fracture turning is not as significant. No apparent differences are observed among the different Young's modulus contrast cases. Regardless of the Young's modulus contrasts, a fracture turns at a fracture toughness contrast of three to four (turning fracture toughness contrast $K_{I C 1} / K_{I C 2}>3-4$ ) in the lower principal stress difference cases ( $\Delta \sigma<=1.0 \mathrm{MPa}$ ) and at eight to ten fracture toughness contrast in the higher principal stress difference cases $(\Delta \sigma \square 20 \mathrm{MPa})$. Please note that the same kind of results are obtained in the fracture propagations from the harder layer to the softer layer though they are not shown in this paper (to avoid redundancy). The effect of Young's modulus contrast on fracture turning is not significant also in fracture propagations from the harder layer to the softer layer.

The tendency that the fracture turning criteria depends less on Young's modulus contrast is partially explained by using the contrast of the critical force scalar-states near the tip between the horizontal bond in Layer 1 and the vertical bond across the layer interface which may be used as the turning criteria. From Equation (26), and (27), the critical force scalar-state for the horizontal bonds in Layer 1 and the critical force scalar-state for the vertical bonds across the layer interface are approximated as,

$$
\begin{aligned}
\underline{t}^{*}[\mathbf{x}, t]\langle\xi\rangle_{\text {crit_hori }} & =\underline{t}[\mathbf{x}, t]\langle\xi\rangle_{\text {crit_hori }}-t_{0}[\mathbf{x}]\langle\xi\rangle_{\text {hori }} \\
& =\frac{24 E_{1}}{5 \pi r \delta^{3}}\|\boldsymbol{\eta}\|_{\text {crit_hori }}-\frac{3 \sigma_{H}}{\pi \delta^{3}} \\
& =\frac{24 E_{1}}{5 \pi r \delta^{3}} \frac{5 \sqrt{r}}{8 E_{1}}\left(\sqrt{\frac{9 \pi}{8}} K_{I C 1}+\sqrt{r} \sigma_{H}\right)-\frac{3 \sigma_{H}}{\pi \delta^{3}} \\
= & \frac{9}{2 \sqrt{2 \pi r} \delta^{3}} K_{I C 1} \\
\underline{t}^{*}[\mathbf{x}, t]\langle\xi\rangle_{\text {crit_vert }} & =\frac{t[\mathbf{x}, t]\langle\xi\rangle_{\text {crit_hori }}-t_{0}[\mathbf{x}]\langle\xi\rangle_{\text {vert }}}{}=\frac{24 \bar{E}}{5 \pi r \delta^{3}}\|\eta\|_{\text {crit_vert }}-\frac{3 \sigma_{V}}{\pi \delta^{3}} \\
& =\frac{24 \bar{E}}{5 \pi r \delta^{3}} \frac{5 \sqrt{r}}{8 \bar{E}}\left(\sqrt{\frac{9 \pi \bar{E}}{8 E_{2}}} K_{I C 2}+\sqrt{r} \sigma_{V}\right)-\frac{3 \sigma_{V}}{\pi \delta^{3}} \\
& =\frac{9}{2 \sqrt{2 \pi r} \delta^{3}} K_{I C 2} \sqrt{\frac{\bar{E}}{E_{2}}}
\end{aligned}
$$

From Equation (28) and (29), the ratio of the critical force vector-states between the horizontal and vertical direction is given as follows (note that we assume that the bond distance $r$ is the same in both directions), 


$$
\underline{t}^{*}[\mathbf{x}, t]\langle\xi\rangle_{\text {crit_hori }} / \underline{t}^{*}[\mathbf{x}, t]\langle\xi\rangle_{\text {crit_vert }}=\frac{K_{I C 1}}{K_{I C 2}} \sqrt{\frac{E_{2}}{\bar{E}}}
$$

As shown in Equation (30), the contrast of the critical force scalar-states between the horizontal bond in Layer 1 and the vertical bond across the layer interface is mainly affected by the fracture toughness contrast. The effect of Young's modulus contrast $\left(\sqrt{\frac{E_{2}}{\bar{E}}}\right.$ ) is relatively limited from 0.63 to 1.25 in the ranges where $\mathrm{E}_{1}=10-40 \mathrm{GPa}$, $E_{2}=10-40 \mathrm{GPa}$. Hence, the fracture turning behavior is mainly controlled by the fracture toughness contrast.

\section{(4) Effect of Layer Dipping on Turning}

As shown in Fig. 5 and Fig. 6, for dipping layers, the minimum fracture toughness contrasts for which fracture turning occurs mainly depends on the principal stress difference. It is not affected much by the Young's modulus contrast. This is the same tendency as when the layer dip angle is 0 ("0 degree cases"). The main difference between the 0 degree layer dip cases and the dipping bedding planes is that the fractures only turn updip along the layer interface in the dip angle cases since the updip side is less compressive due to the layer inclination. As shown in Fig. 5, in the cases where the layer dip angle is 15 degree ("15 degree cases"), since the initial effective stress acting on the layer interface in the 15 degree cases is almost the same as the 0 degree cases, the fracture turning criterion is almost the same as the 0 degree cases regardless of the Young's modulus contrast. The minimum fracture toughness contrast for fracture turning is around 3.0 - 4.0 (1.5 MPa m $\mathrm{m}^{0.5} / 0.5 \mathrm{MPa} \mathrm{m} \mathrm{m}^{0.5}-2.0 \mathrm{MPa} \mathrm{m}^{0.5} / 0.5$ $\left.\mathrm{MPa} \mathrm{m}^{0.5}\right)$ in the lower principal stress difference cases $(\Delta \sigma<1.0 \mathrm{MPa})$ and around $8.0-10.0$ in the high principal stress difference cases ( $\Delta \sigma=20.0 \mathrm{MPa}$ ). As shown in Fig. 6 , in the cases where the layer dip angle is 30 degrees (30 degree cases), the minimum fracture toughness contrast for fracture turning becomes lower than for the low layer dip angle cases (the 0 degree cases and the 15 degree cases). For a 30 degree layer dip angle, the minimum fracture toughness contrast for fracture turning is around 3.0 - 4.0 (1.5 $\mathrm{MPa} \mathrm{m}{ }^{0.5} / 0.5 \mathrm{MPa} \mathrm{m}{ }^{0.5}-2.0$ $\mathrm{MPa} \mathrm{m}^{0.5} / 0.5 \mathrm{MPa} \mathrm{m}{ }^{0.5}$ ) for a low principal stress difference $(\Delta \sigma<1.0 \mathrm{MPa})$ and around $5.0-6.0$ for a high principal stress difference condition ( $\Delta \sigma=20.0 \mathrm{MPa}$ ). Since the initial normal effective stress acting on the layer interface in the 30 degree cases is smaller than the 0 degree cases, especially in the high principal stress condition, the fracture propagation along the layer interface is easier than for the low dip angle cases. However, as mentioned in Section 4.1.2.1 (2), a fracture toughness contrast of the order of 5.0 - 6.0 is still extremely high. Judging from the minimum fracture toughness contrast obtained in this simulation study, no fracture turning is expected in a deep reservoir even if the layer interface is highly inclined.

\subsubsection{Kinking}

\section{(1) Mechanism}

As shown in Fig. 5 and Fig. 6, if the layer interface is inclined, the fractures do not go straight but kink before they reach the layer interface in many cases. Here, we explain the basic mechanism of "kinking" and the important parameters that control fracture kinking. 
Fig. 9 shows the distribution of horizontal stress and damage at different time steps in the case where the dip angle is 15 degrees, the Young's modulus contrast is 4.0, and the principal stress difference is $1.0 \mathrm{MPa}$. As shown in Fig. 9, if the dip angle is not zero and the Young's modulus contrast is more than 1.0, the fracture turns to the right before it hits the layer interface. Since the displacement of Layer 2 is prevented more by the smaller displacement of Layer 1 to the left of the fracture, the crack propagation becomes relatively difficult on the left side, which results in the fracture turning toward the right. Note that if the fractures propagate from the harder layer to the softer layer, the fracture turns to the left instead of right since the direction perpendicular to the layer interface is the easiest direction for fracture propagation. These cases are shown in Section 4.2.

\section{(2) Effect of Young's modulus contrast on kinking}

Fig. 10 shows the damage distribution just before crossing the layer interface for the different Young's modulus contrast cases. In these cases, the other parameters are fixed as follows (layer dip angle $=15$ degree, the fracture toughness contrast $=K_{I C 1} / K_{I C 2}=1.4 / 0.5$, and the principal stress difference $=1.0 \mathrm{MPa}$ ). As shown in Fig. 10, since the fracture turning is caused by the difference of the displacement between Layer 1 and Layer 2, a higher Young's modulus contrast causes more fracture turning. As shown in Fig. 5 (a) and Fig. 10 (a), if the fracture toughness contrast is 1.0 and the layer dip angle is 15 degrees, no fracture turning is expected before the fracture reaches the layer interface. However, as shown in Fig. 6 (a), if the layer dip angle is 30 degrees, the kinking region appears even if the fracture toughness contrast is 1.0. In these cases, there is no kinking before the fracture reaches the layer interface but the kinking occurs along the layer interface due to the fracture toughness contrast.

\section{(3) Effect of fracture toughness contrast on kinking}

Fig. 11 shows the damage distribution just before the fracture crosses the layer interface for the different fracture toughness contrast cases. In these cases, the other parameters are fixed as follows (layer dip angle $=15$ degree, the Young's modulus contrast $=E_{1} / E_{2}=40 / 10$, and the principal stress difference $=1.0 \mathrm{MPa}$ ). As shown in Fig. 11, the degree of fracture kinking (kinking angle) is not different for the different fracture toughness contrast cases. Since the kinking is caused by the displacement difference between the two layers under compression, the parameters which are not directly related to the compressional displacement such as fracture toughness do not affect the degree of kinking.

\section{(4) Effect of principal stress difference on kinking}

Fig. 12 shows the damage distribution just before the fracture crosses the layer interface for the different principal stress difference cases. In these cases, the other parameters are fixed as follows (layer dip angle $=15$ degree, the Young's modulus contrast $=E_{1} / E_{2}=40 / 10$, and the fracture toughness contrast = $K_{I C 1} / K_{I C 2}=1.4 / 0.5$ ). As shown in Fig. 12, the more the principal stress difference is, the less the fracture turns before it reaches the layer interface. Since the larger vertical stress makes the bonds in the vertical direction have a higher compressional stress than the bonds in the horizontal direction, breaking the vertical bonds becomes more 
difficult. Therefore, the fracture turning into the horizontal direction is more difficult in the larger principal stress difference case.

\section{(5) Effect of layer dip angle on kinking}

Fig. 13 shows how the fracture turning angle is different depending on the layer dip angle. As shown in Fig. 13, the fractures turn more in the cases where the layer dip angle is 30 degrees than the cases where the layer dip angle is 15 degrees. In the larger dip angle cases, since the horizontal displacement in Layer2 is more influenced by Layer 1 at the left of the fracture, the fracture propagates more toward the right.

\subsubsection{Branching}

As shown in Fig. 4 (d), our results show that if the following conditions are satisfied, the fracture branches at the layer interface (first turns along the layer interface and then quickly move into the first layer).

- Fracture propagates from the low Young's modulus layer to the high Young's modulus layer

- Young's modulus contrast between the two layers is high $\left(E_{1} / E_{2} \geq 8.0\right)$.

- Fracture toughness contrast is less than $1.0\left(K_{I C 1} / K_{I C 2}<=1.0\right)$.

In essence, if the upper layer material is very brittle (High Young's modulus and Low Fracture toughness), fracture branching will occur. Fig. 14 shows the damage distribution near the fracture tip in the branching case where the Young's modulus contrast, fracture toughness contrast, and principal stress difference are $8\left(E_{1} / E_{2}=\right.$ 80GPa/10GPa), 1.0 (=0.5 MPa m $\left.{ }^{0.5} / 0.5 \mathrm{MPa} \mathrm{m}^{0.5}\right)$, and 1.0 MPa respectively. As shown in Fig. 14, if Layer 1 is much more brittle than Layer 2 as mentioned above, the damage zone begins to grow before the fracture tip reaches the layer interface due to the much smaller energy requirement for the bond breakage in Layer 1 than for Layer2. In this case, the energy required for breaking bonds in Layer 1 is just $12.5 \%$ of the energy required to break bonds in Layer 2. Hence, even small deformations, induced by the fracture propagation in Layer2, which do not break bonds in Layer 2 can break the bonds near the layer interface and in Layer 1. As shown in Fig. 14 (c), since Layer 1 has already been damaged when the fracture reaches the layer interface, the fracture continues to propagate through the pre-damaged zone in Layer 1 . Note that the fracture perfectly branches in this example case due to the perfect symmetry of the mechanical properties in front of the fracture tip. However, for most cases, the mechanical properties in front of the fracture tip are not fully symmetric at the layer interface. For such cases, fracture "kinking" may occur (in other words, one side of the branch may grow) instead of "branching" since the fracture always propagates through the weakest path (the easiest open path).

\subsubsection{Effect of Layer Thickness}

In order to investigate how the layer thickness affects the fracture propagation near the layer interface, as shown in Fig. 15, we prepared three different models by changing the $2^{\text {nd }}$ layer thickness for two different layer dip angles (0 degrees and 30 degrees). Then we selected the following reference cases (please see Table 4) from the previous cases shown in Fig. 4 (c) and Fig. 6 (c) and simulated fracture propagation by changing the $2^{\text {nd }}$ layer thickness for each of the reference cases. 
Fig. 16 and Fig. 17 show the results for fracture propagation for the different layer thickness cases. As shown in these figures, the thinner the $2^{\text {nd }}$ layer thickness is, the higher the minimum fracture toughness contrast needed for fracture turning. These result clearly shows that not only the mechanical properties of the interface layers but also those of other layers could affect the fracture propagation behavior at the target layer interface in accordance with the distance from the layer interface. Fig. 18 shows the horizontal stress difference between the thinnest layer case $(1=1.2 \mathrm{~cm})$ and the reference case $(l=10.0 \mathrm{~cm})$ in the 0 degree case. As shown in Fig. 18 , if the $2^{\text {nd }}$ layer thickness is thin, since the existence of the softer layer (the $1^{\text {st }}$ layer) makes the displacement of the $2^{\text {nd }}$ layer larger, the stress reduction near the layer interface between the $2^{\text {nd }}$ layer and the $3^{\text {rd }}$ layer is bigger for the thinnest $2^{\text {nd }}$ layer thickness case than for the reference case. This leads to easier fracture penetration in the $2^{\text {nd }}$ layer in the thinnest layer cases. As shown in Fig. 16, in the 0 degree case, if the 2nd layer thickness is more than $4.8 \mathrm{~cm}$, the fracture turning criteria is the same as the reference case regardless of the principal stress difference. However, in the 30 degree case, if the principal stress difference is high, the fracture turning behavior is still different from the reference case even in the case where the $2^{\text {nd }}$ layer thickness is $4.8 \mathrm{~cm}$. On the other hand, if the principal stress is low, the fracture propagation behavior is the same as the reference case in the cases where the layer thickness is more than $2.4 \mathrm{~cm}$. These differences may be caused by the kinking angle difference between the two different cases. Since the fracture has already turned before reaching the layer interface in the low principal stress difference case, the horizontal stress reduction near the fracture tip in the $2^{\text {nd }}$ layer is not as large as in the high principal stress difference case. Therefore, if the principal stress difference is low, the layer thickness difference does not significantly affect the fracture propagation behavior in the 30 degree cases. Note that, in the 30 degree cases, as shown in Fig. 17, regardless of the $2^{\text {nd }}$ layer thick ness, the magnitudes of fracture turning before the fracture reaches the layer interface are almost same even for the thinnest $2^{\text {nd }}$ layer thickness case. These results suggest that fractures can frequently kink in most reservoirs which have some degree of bed dipping and vertical heterogeneity in mechanical properties. The examples of frequent kinking in vertically heterogeneous inclined reservoir are shown in the section 4.2 .

\subsubsection{Effect of Horizontal Stress Difference}

For investigating the effect of horizontal principal stress difference between the layers, we choose the eight reference cases from the previous cases shown in Fig. 4 (two cases for each Young's modulus contrast). All these reference cases have the same layer dip angle ( $\theta=0$ degree), the same principal stress difference ( $\Delta \sigma=10 \mathrm{MPa})$, the same horizontal principal stress both in Layer 1 and Layer 2 ( $\sigma_{H}=40.0 \mathrm{MPa}$ ), and a smaller fracture toughness contrast than the minimum fracture toughness contrast for fracture turning. As shown in Fig. 19, by applying a $5 \mathrm{MPa}$ higher effective horizontal principal stress to Layer 1 than in the reference cases, we simulate how fracture turning criterion changes as the horizontal stress is increased. Table 5 shows the reference cases for the simulations in this section.

Fig. 20 shows a summary of the simulation results in this section. As shown in Fig. 20, the fractures turn at a smaller fracture toughness value than the reference cases (in every instance). These results show that increasing the horizontal principal stress difference between layers lowers the minimum fracture toughness contrast for fracture turning. However, when we consider the fact that a large horizontal stress difference is only expected in 
deep reservoirs where the required fracture toughness contrast for the fracture turning is around eight to ten, the effect of horizontal principal stress difference (the reduction of the minimum fracture toughness change order of 1.0 - 2.0) may not significantly affect the fracture turning behavior (practically turning is not caused only by horizontal stress difference).

\subsubsection{Effect of Weak Surface}

In the previous sections, we investigated the effect of several mechanical properties based on the assumption that layer interface is fully bonded (in other words, the layer interface is not allowed to be damaged). However, layer interfaces are not always strongly bonded. Sometimes, they can be weakly bonded and easily slip along the layer interface. In order to analyze the effect of a weak layer interface on fracture propagation (turning, kinking and branching), we apply the following shear failure parameter ( the layer interface in the cases shown in Fig. 4, Fig. 5, and Fig. 6 ("original cases"), and simulate fracture propagation in these cases ("weak surface cases"). Note that, in these cases, all the parameters except the shear failure parameters are the same as the original cases.

Fig. 21 - Fig. 23 show the fracture propagation behavior near the layer interface for the different layer dip angle cases ( 0 degree, 15 degree, and 30 degree cases in the weak surface cases respectively). As shown in these figures, the minimum fracture toughness contrasts for fracture turning in the weak surface cases are lower than the original cases for all layer dip angles. However, the degree and the criteria for fracture turning are very different depending on the layer dip angles. As shown in Fig. 21, in the 0 degree cases, even if the cohesion of the layer interface is zero, the difference of the minimum fracture toughness contrast for fracture turning between the original cases and the weak surface cases is less than 1.0. Since the initial normal stress acting on the layer interface is equivalent to the maximum principal stress in these cases, shear failure along the layer interface is difficult and unlikely to occur. These results show that fracture turning is not expected in deep reservoirs even if the layer interface is weak. Note that fracture branching is not observed in the low fracture toughness region in the highest Young's modulus case (see Fig. 21 (d)). Since the part of the bonds across the layer interface break in shear failure due to the low shear failure criteria, the displacement of the softer layer cannot fully transfer to the harder layer in these cases. Shear slip prevents fracture branching in the highest Young's modulus contrast case.

As shown in Fig. 22, in the 15 degree cases, the differences of the minimum fracture toughness contrast for fracture turning between the original cases and the weak surface cases are larger than the 0 degree cases since shear failure is more likely to occur along the layer interface. A 1.5 to 2.0 reduction in the fracture toughness contrast is observed in these cases. Note that, as shown in Fig. 22, the extent of fracture kinking is smaller in the weak surface cases than in the original cases. In these cases, since Layer 2 can slip along the layer interface, the displacement of Layer 2 is not limited by Layer 1, which results in more straight fracture propagation into Layer 2.

As shown in Fig. 23, in the 30 degree cases, the fractures always turn along the layer interface regardless of the principal stress difference and the fracture toughness contrast since shear failure always occurs along the layer interface. A weak layer interface has a significant influence on fracture turning in high dip angle layers. These 
results show that if the layer is highly inclined and the layer interface is not fully bonded, turning can occur even in the deep reservoir.

\subsection{Investigation of the fracture propagation behavior in multiple layer cases}

In shale gas reservoirs, mechanical property distribution in vertical direction is highly heterogeneous. As Passey et al. (Passey et al., 2010) point out, mineralogical changes in shale gas reservoirs can be observed at the order of $\mathrm{mm}$ and $\mathrm{cm}$ scale as well as meter scale (Fig. 24).

In this section we investigate how fracture propagation is affected if this kind of small scale heterogeneity (cm scale heterogeneity) exists in the reservoir. We prepare the two types of models ("Model 1" and "Model 2") which have a $30 \mathrm{~cm} \times 30 \mathrm{~cm}$ model domain and consist of $150 \times 150$ elements. Both models are divided into multiple layers which have constant thickness $(=2 \mathrm{~cm})$. The layer dip angle of Model 1 is 0 degrees, while the layer dip angle for Model 2 is 30 degrees. As shown in Fig. 25, in order to represent small scale vertical heterogeneity, we alternatively assign two different sets of mechanical properties to each layer in both models. As shown in Fig. 26, to investigate the effect of initial stress distribution, one of two different stress initialization methods is applied in each case (Initialization 1 or Initialization 2).

- Initialization 1 (assuming homogeneous stress distribution): At time $t<0$, a constant horizontal stress $\left(\sigma_{H}=40 \mathrm{MPa}\right)$ and vertical stress $\left(\sigma_{V}=60 \mathrm{MPa}\right)$ are assigned to each element through the background stress tensor method.

- Initialization 2 (assuming zero strain in horizontal direction): At time $\mathrm{t}<0$, a roller boundary condition is applied to the side boundaries (no deformation in the horizontal direction) and only a normal traction of magnitude ( $\sigma_{V}=60 \mathrm{MPa}$ ) is applied to the top and the bottom boundary. At time $\mathrm{t}>=0$, the roller boundary condition applied to the side boundaries is replaced by constant traction boundaries which gives the same traction as the roller boundary condition in order to allow horizontal displacement of the side boundaries due to fracture propagation.

After stress initialization (at time $\mathrm{t}>=0$ ), fluid is injected from the "injection dual point" at the center of the model domain at constant rate to propagate a fracture. We investigated the effect of small scale heterogeneity on fracture propagation by changing the contrast in mechanical properties and the initial stress distribution in both models. Table 6 shows the case settings. Table 7 shows the common calculation settings for these cases. In these cases, we assumed that the energy release rate is the same both in the higher Young's modulus layer and the smaller Young's modulus layer (except in the contrast-high 2 case and contrast-high 2_dip case). Fig. 27 shows the initial stress distribution in the cases where the Initialization 2 method is applied. Note that in Model 1 (layer dipping angle = 0), we only apply Initialization1 to all the cases. Since the same Poisson's ratio is applied in every layer in these cases, even if Initialization 2 is applied, it also gives the same initial stress distribution as Initialization 1 in Model 1.

Error! Reference source not found. - Fig. 30 show the mechanical and fluid property distributions after fracture propagation (note that the displacements are 50 times exaggerated in these figures.) As shown in Error! 
Reference source not found. (A) and Fig. 28 (B), in the cases where the layer dip angle is zero and the Young's modulus contrast is not very high (less than 4.0), the fractures just propagate in the maximum principal stress direction even if the vertical heterogeneity exists in the reservoir. However, as shown in Fig. 28 (C), in the cases where the layer dip angle is zero and the Young's modulus contrast is very high $(=8.0)$, the fracture propagation is affected by the small scale heterogeneity in the reservoir. In this case, the fracture does not go straight, but branches at the layer interface when it propagates from the lower Young's modulus layer (softer layer) to the higher Young's modulus layer (harder layer) due to the pre-damage zone in the higher Young's modulus layer. As explained in section 4.1.2.3, if the Young's modulus between the two layer is highly different, the required displacement for the fracture propagation in the harder layer is much smaller than the softer layer (in this case, the critical displacement of the softer layer is about one-third of the harder layer). Therefore, the displacement induced by the fracture propagation in the softer layer can cause damage in the harder layer before the fracture tip reaches the layer interface, which finally causes fracture branching. As shown in Fig. 28 (D), if the harder layer's energy release rate is smaller than the softer layer (high_contrast 2 case: $G_{E_{-} \text {hard }} / G_{E_{-} s o f t}=0.25$ ), branching is more clearly observed due to the higher critical displacement contrast between the harder layer and the softer layer. These high Young's modulus contrast cases demonstrate that even a thin (order $1 \mathrm{~cm}$ ) high Young's modulus layer such as a calcite vein can cause fracture branching at the layer interface between the softer layer and the harder layer. When we take into account the published mechanical property of calcite $\left(\mathrm{E}=84.3 \mathrm{GPa}, \mathrm{K}_{\mathrm{IC}}=\right.$ 0.19 MPa $\mathrm{m}^{0.5}$ )(Atkinson, 1987; Gray Mavko, 2009), branching may occur in some reservoirs where calcite veins exist. Note that, due to the difficulty of convergence of the calculation when the fracture tips reach the pre-damage zone in the harder layer, the symmetricity of the fracture propagation is not perfectly kept in these high Young's modulus contrast cases. However, since the pre-damage zone in the harder layer has already existed before the fracture tip reaches the layer interface, branching in these cases is not the result of numerical instability.

If the layer has a high dip angle (30 degrees), regardless of the initial stress distribution (Initialization 1, assuming a homogeneous stress distribution) and Initialization 2 (assuming zero strain in the horizontal direction)), the fracture kinks at the layer interface even in the low Young's modulus contrast case ( $E_{\text {hard }} / E_{\text {soft }}=2.0$ ) since the fracture propagates in the propagation direction offering the least resistance. As shown in Fig. 29 (A) and Fig. 30 (A), in the dipping layers, the fracture turns as if avoiding the harder layer and staying in the softer layer. In addition, the fracture also turns towards the softer layer when propagating in the harder layer. Due to the combination of these two types of kinking in the softer layer and the harder layer, the total kinking angle at the layer interface finally becomes more than 60 degrees in these cases. When the two different stress initializations are compared, Initialization 2 which has a higher horizontal stress contrast between the high Young's modulus layer and the low Young's modulus layer, the fracture is more likely to turn in the "low_contrast_dip2" case (non-homogeneous stress distribution case) than the "low_contrast_dip" case (homogeneous stress distribution case). The stress difference caused by mechanical property variations enhances fracture turning. As shown in Fig. 29 (B) and Fig. 30 (B), in the cases where the Young's modulus contrast is not 
as large ( $E_{\text {hard }} / E_{\text {soft }}=4.0$ ), the kinking angle of the fracture at the layer interface becomes higher (more than 70 -80 degrees) than the lower Young's modulus contrast cases. The fracture turning angle in the non-homogeneous stress distribution case (middle_contrast_dip2 case) is higher than the homogeneous stress distribution case (middle_contrast_dip case). In the cases where the Young's modulus contrast is the highest ( $E_{\text {hard }} / E_{\text {soft }}=8.0$ ), as shown in Fig. 29 (C) and Fig. 30 (C), the fracture kinks at around 80 - 90 degrees at the layer interface in both stress distribution cases. These layer dipping cases clearly show the tendency that the higher the Young's modulus contrast, the more local kinking is expected in a dipping layer. When compared with the two different stress initialization cases, the overall fracture propagation direction in the homogeneous initial stress distribution case is about 12 degrees deviated from the maximum principal stress direction (Fig. 29 (C)) while the overall fracture propagation direction in the non-homogeneous initial stress distribution case is almost parallel to the maximum principal stress direction (Fig. 30 (C)). As shown in Fig. 29 (D) and Fig. 30 (D), if the energy release rate in the harder layer is $25 \%$ of the softer layer in the highest Young's modulus contrast cases, the fracture propagation geometries in the different stress initialization cases (high_contrast2_dip case and high_contrast2_dip case) also show very high turning angle ( $80-90$ degrees). However, the global fracture propagation direction in the uniform initial stress distribution case (high_contrast2_dip case) is different from the non-uniform initial stress distribution case (high_contrast2_dip2 case). In the "high_contrast2_dip" case, as shown in Fig. 31, one observes the same kind of pre-damaged zone, as in the case where the layer dip angle is zero and the other conditions are the same. However, due to the layer dipping angle, the magnitude of the two pre-damage zones ahead of the fracture tip is not equivalent. One of the pre-damage zones which is closer to the principal stress direction in the softer layer grows more than the other side of the pre-damage zone due to the ease of fracture propagation. Therefore, the fracture preferentially selects the larger pre-damaged zone and the overall fracture propagation direction in this case is different from the "high_contrast_dip" case. In the non-uniform stress distribution case (high_contrast2_dip2 case), the pre-damage zones do not appear in the high Young's modulus layers due to the high stress concentration in the high Young's modulus layers. Therefore, the global fracture propagation direction is still same as the high_contrast_dip2 case even if the fracture toughness in the high Young's modulus layer is low.

These simulation results clearly show that, if the layer interface is inclined, the effect of $\mathrm{cm}$ scale sublayers on fracture propagation cannot be neglected. As Fisher et al. point out (Fisher and Warpinski, 2012), kinking at the layer interface will affect proppant transport (screen out could occur) due to changes in the fracture width at the kinking point. In addition, the frequent kinking could also have a significant influence on the net pressure (fracturing fluid pressure distribution in the frequently kinked fracture geometry may be totally different from a single planar fracture). Judging from the kinking angle, even if the Young's modulus contrast is low, inclined sub-layers could be an obstacle to fluid flow, fracture growth and proppant transport. 


\section{Conclusion}

We systematically investigated fracture propagation in heterogeneous reservoirs by using different geological models with different types of heterogeneity (two layer model, three layer model, multi-layer model, and arbitrary micro-scale heterogeneity).

1. In the two layer model, we observed three characteristic fracture propagation behaviors near the layer interface: "turning", "kinking", and "branching". The following conclusions were drawn from this study.

\section{Fracture Turning}

- If the layer interface is not damaged, fracture turning along the layer interface is primarily controlled by the fracture toughness contrast and the principal stress difference. A lower principal stress difference and a higher toughness contrast facilitates fracture turning along the layer interface. The effect of other parameters (Young's modulus contrast, layer dip angle, and horizontal stress contrast between layers) on turning is limited.

- If the layer interface is damaged (weak), the fracture always turns at high interface dip angles ( $\theta>=30$ degree) regardless of the toughness contrast between layers or the principal stress difference. However, if the interface dip angle is low ( $\theta<=15$ degree), fracture turning is primarily controlled by fracture toughness contrast and principal stress difference.

- Layer thickness also affects fracture turning along the layer interface. The thinner the layer in front of the fracture tip, the easier it is for the fracture to cross the layer interface.

- Based on the required fracture toughness contrast, fracture turning along the layer interface is expected only in shallow reservoirs in which the principal stress contrast is less than $1 \mathrm{MPa}$ or in reservoirs which have highly dipping or weak layer interfaces.

\section{Fracture Kinking}

- If the layer interface is inclined, the propagating fracture kinks before it reaches the layer interface. If the fracture propagates from a softer layer to a harder layer, it turns as if avoiding the layer interface. On the other hand, if the fracture propagates from a harder layer to a softer layer, it turns as if trying to cross the layer interface at a right angle.

- The magnitude of fracture kinking is primarily controlled by the magnitude of Young's modulus contrast and layer dip angle. The effects of fracture toughness contrast and layer thickness on fracture kinking are limited, which suggests that, if layers are inclined enough, the fracture can kink in reservoirs which contain small scale heterogeneities in Young's modulus (bedding planes in the vertical direction) as are commonly observed in shale reservoirs. 


\section{Branching}

- If the Young's modulus contrast is very high $(\mathrm{E} 1 / \mathrm{E} 2>=8.0)$, fracture toughness contrast is low ( $K_{I C 1} / K_{I C 2}<=1.0$ ), and the layer interface is not inclined), a propagating fracture branches in the harder layer due to the damage ahead of the fracture tip in the harder layer.

2. In a multi-layer model, we investigated the effect of many sub-layers or bedding planes on fracture propagation. The following conclusions are obtained.

- If the layers are not inclined, the effect of small scale heterogeneity (sub layers) on fracture geometry is limited. Only in the case where a small vein which has very high Young's modulus and low fracture toughness contrast exists, a fracture can branch in such a layer. The fracture propagation pressure increases as the contrast between the Young's moduli and fracture toughness in the layers increases.

- If the layers are inclined, the fracture can frequently kink at a high angle in the small scale sub layers even if the Young's modulus contrast is low $(<=2.0)$ and the principal stress difference is high. This result suggests that proppant screen out or proppant bridging is likely to happen in a highly inclined, vertically heterogeneous reservoir.

\section{Acknowledgment}

This work is supported by DOE Grant No. DE-FOA-0000724 and by the member companies participating in the Joint Industry Program on Hydraulic Fracturing and Sand Control at the University of Texas at Austin.

\section{Figures}

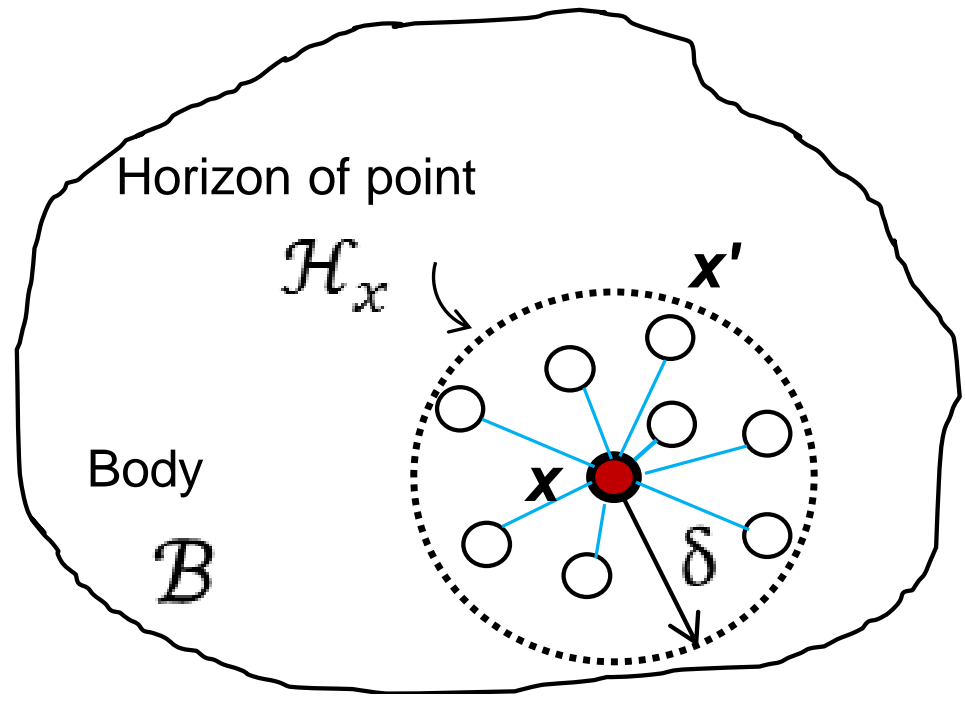

Fig. 1 "Horizon" and "Bond"(Ouchi et al., 2015b). 


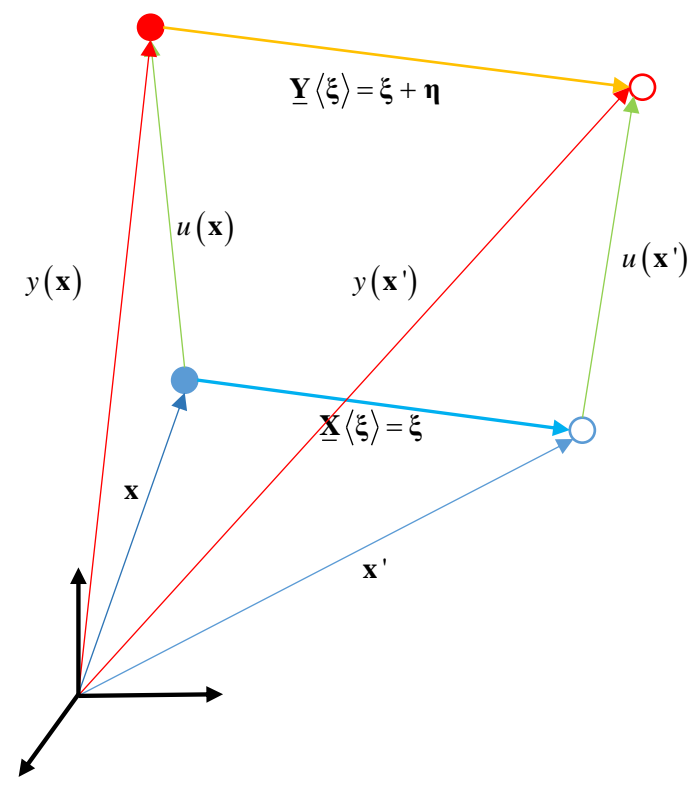

Fig. 2 Reference and deformed configuration.

Extracting a small area near layer boundary
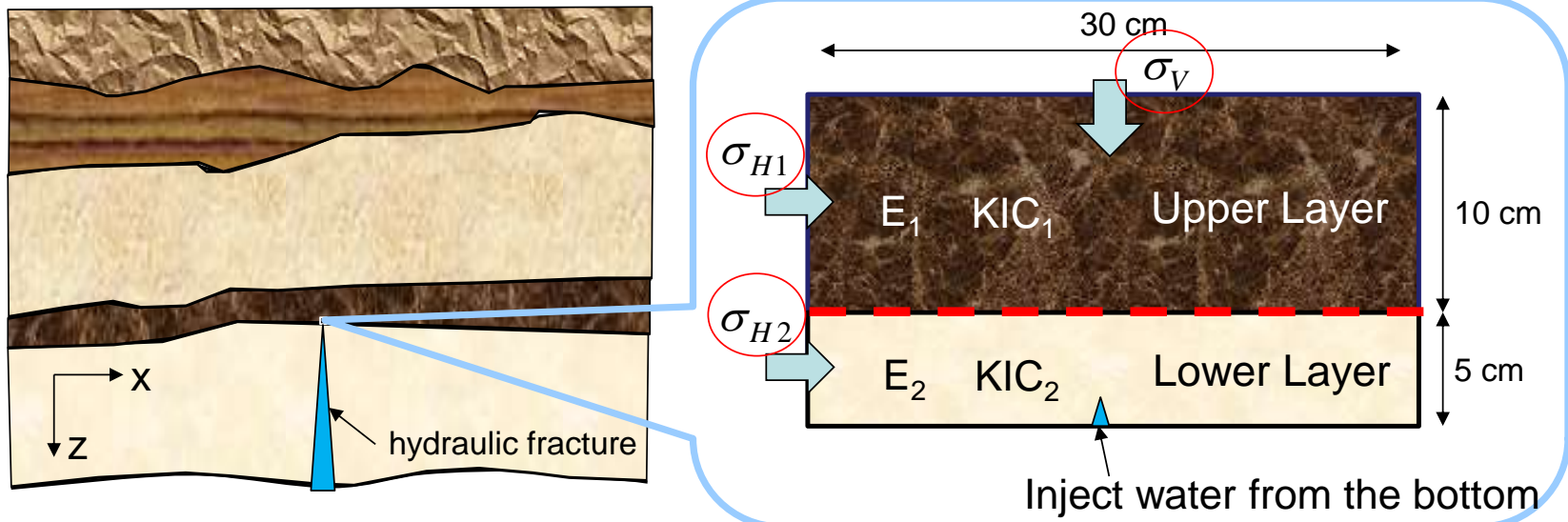

Fig. 3 Schematic view of the 2 layer model. 


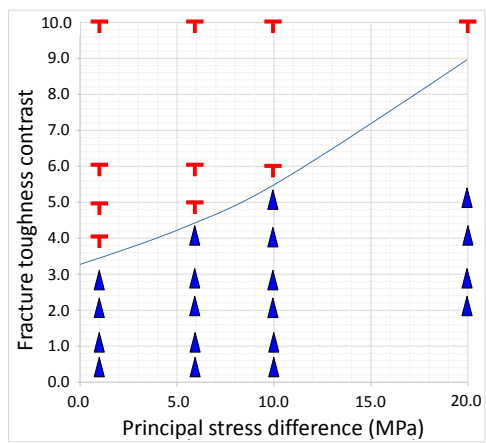

(a) $\mathrm{E}_{1}=10 \mathrm{GPa}$

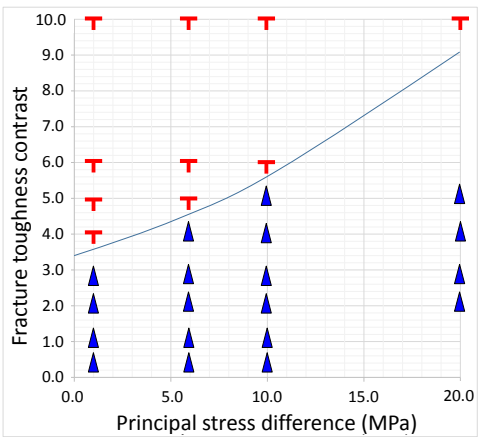

(b) $E_{1}=20 \mathrm{GPa}$

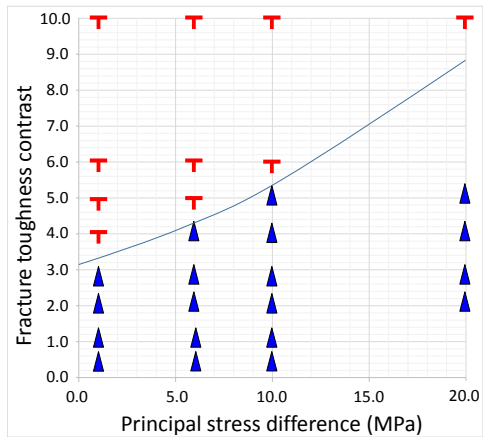

(c) $\mathrm{E} 1=40 \mathrm{GPa}$
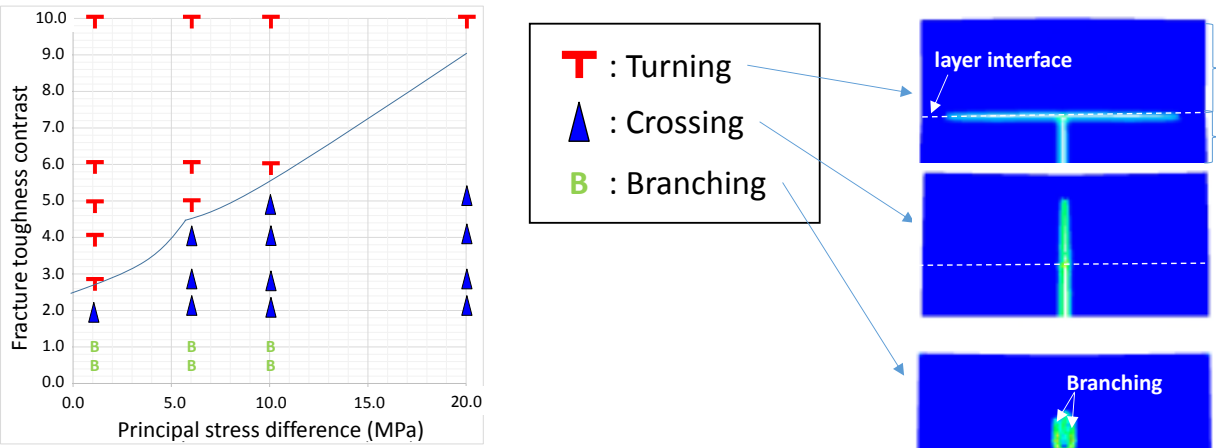

layer 1

(d) $\mathrm{E}_{1}=80 \mathrm{GPa}$

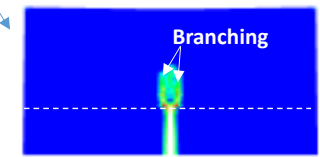

Fig. 4 Fracture turning behavior (layer dip angle $=0$ degee, E2=10 GPa).

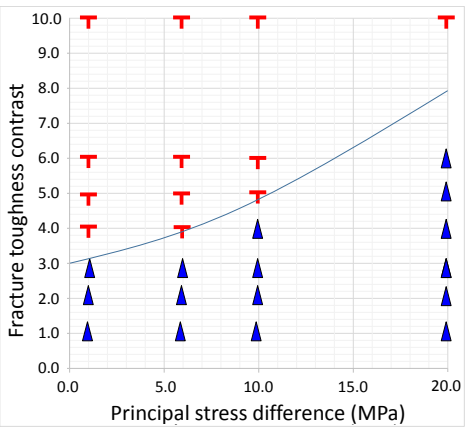

(a) $\mathrm{E}_{1}=10 \mathrm{GPa}$

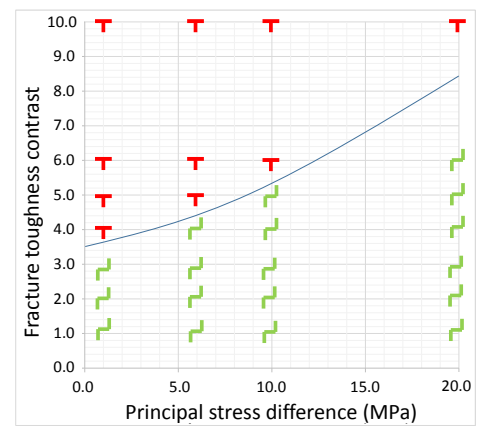

(b) $\mathrm{E}_{1}=20 \mathrm{GPa}$

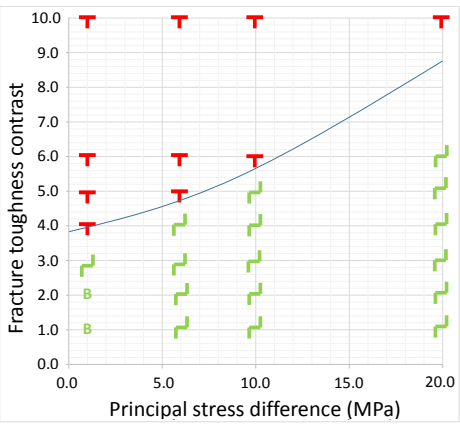

(c) $\mathrm{E}_{1}=40 \mathrm{GPa}$

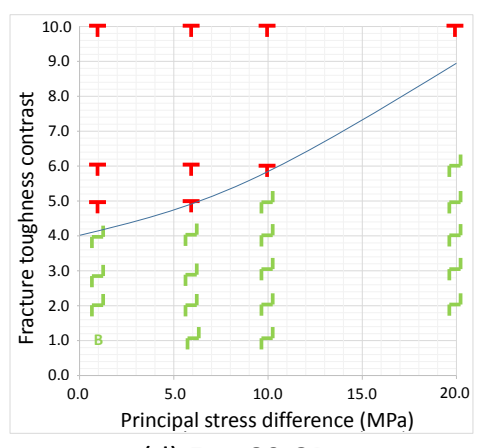

(d) $\mathrm{E}_{1}=80 \mathrm{GPa}$

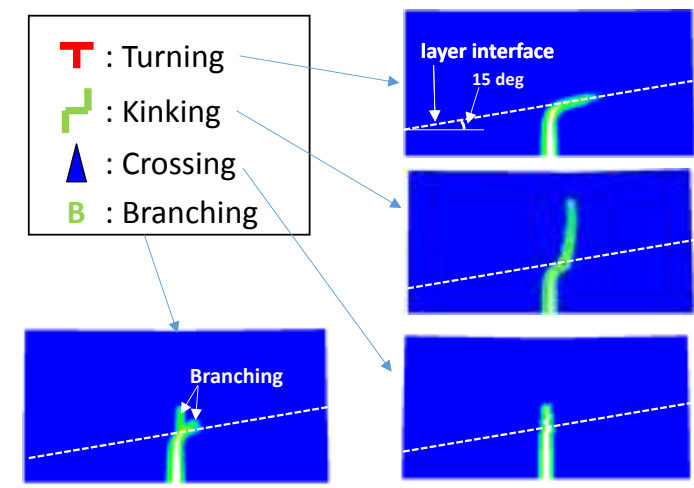

layer 1

layer 2

Fig. 5 Fracture turning behavior (layer dip angle $=15$ degee, E2=10 GPa). 


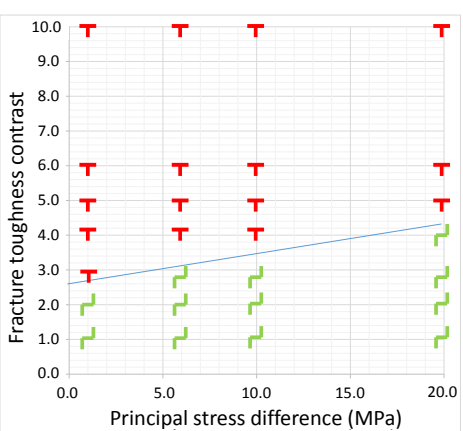

(a) $\mathrm{E} 1=10 \mathrm{GPa}$

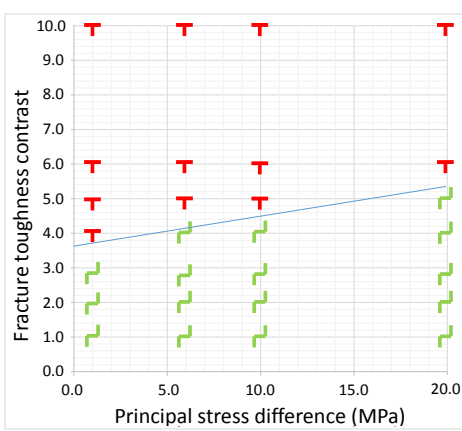

(b) $\mathrm{E} 1=20 \mathrm{GPa}$

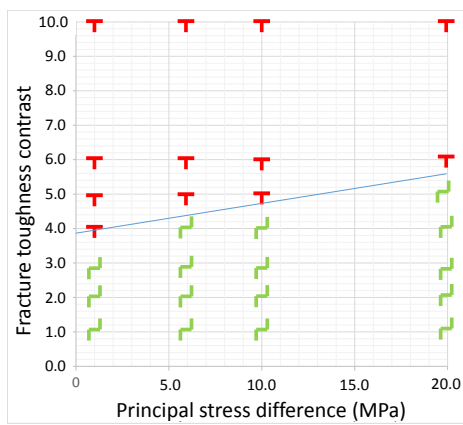

(c) $\mathrm{E}_{1}=40 \mathrm{GPa}$

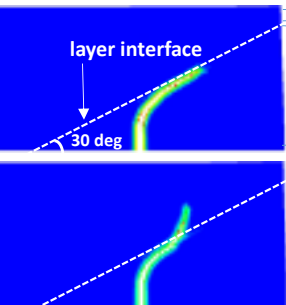

layer 1

layer 2

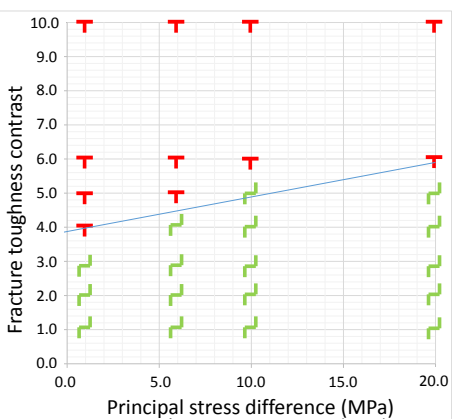

T : Turning II : Kinking

Fig. 6 Fracture turning behavior (layer dip angle $=30$ degree, E2=10 GPa).

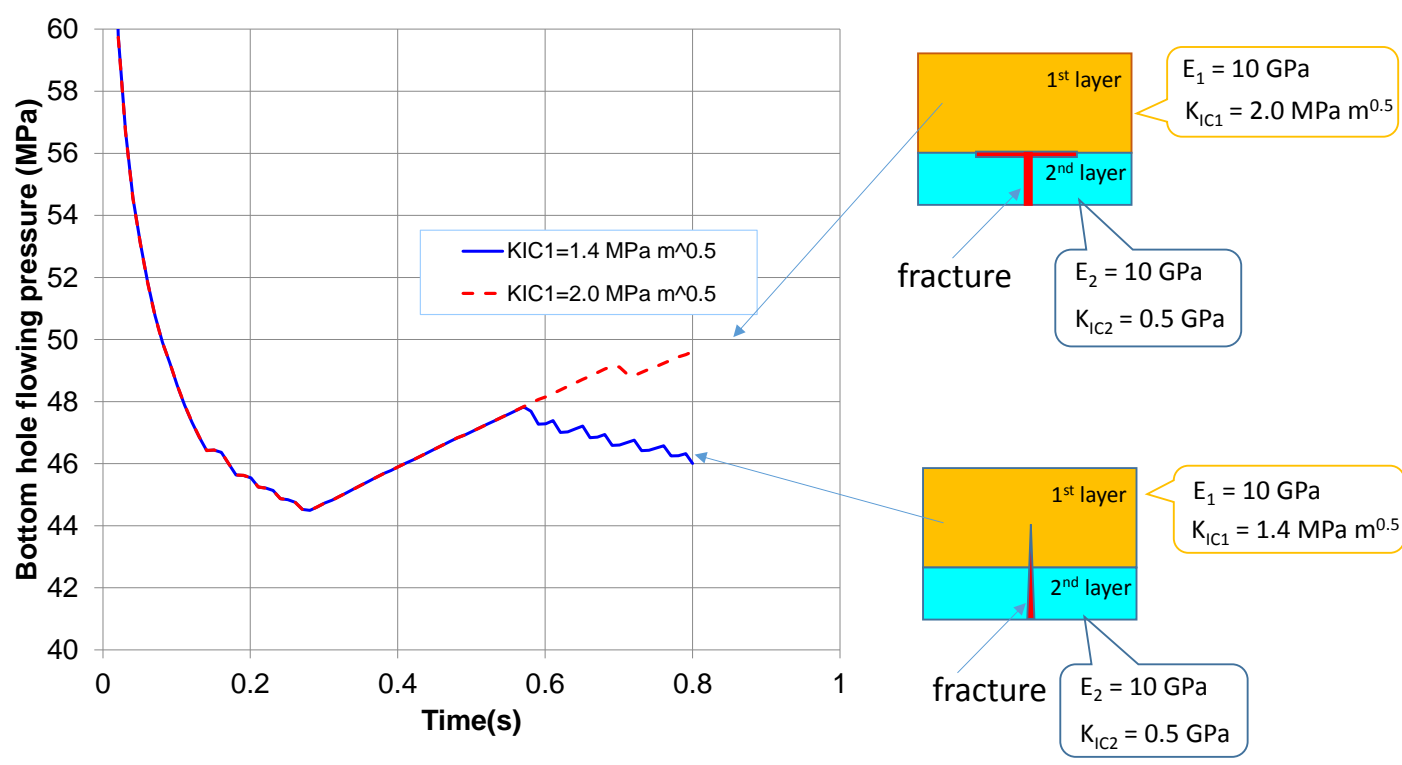

Fig. 7 Bottomhole pressure change with time $(\mathrm{E} 2=10 \mathrm{GPa}$, principal stress difference $=1 \mathrm{MPa})$. 


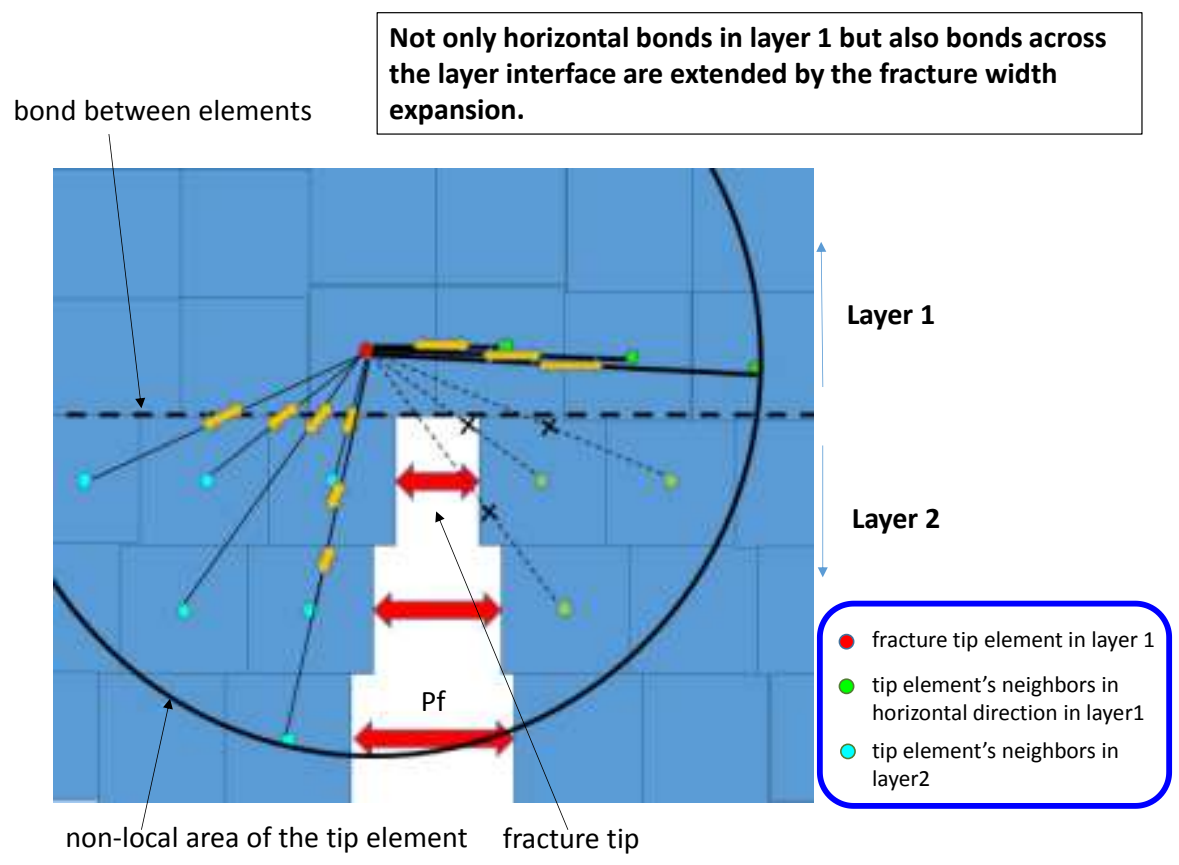

Fig. 8 Schematic view of bond extension near layer interface.

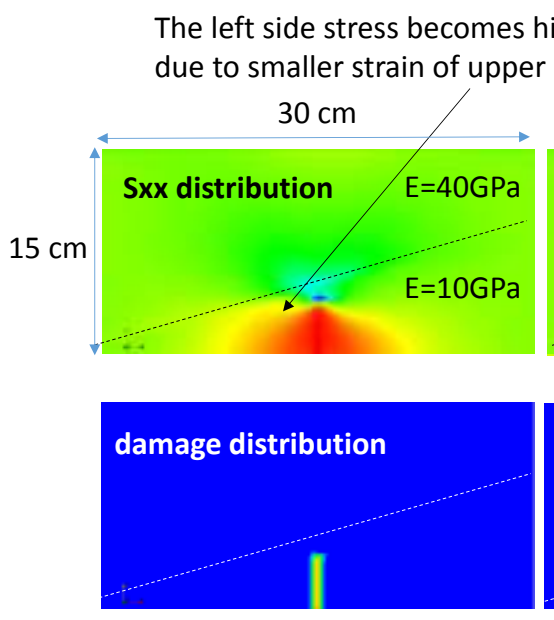

(a) after $0.27 \mathrm{sec}$
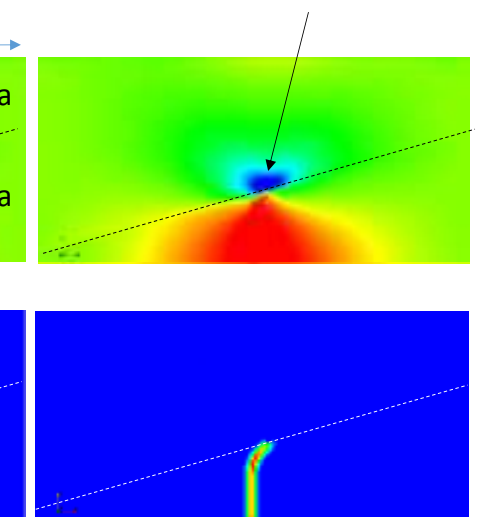

(b) after $0.47 \mathrm{sec}$
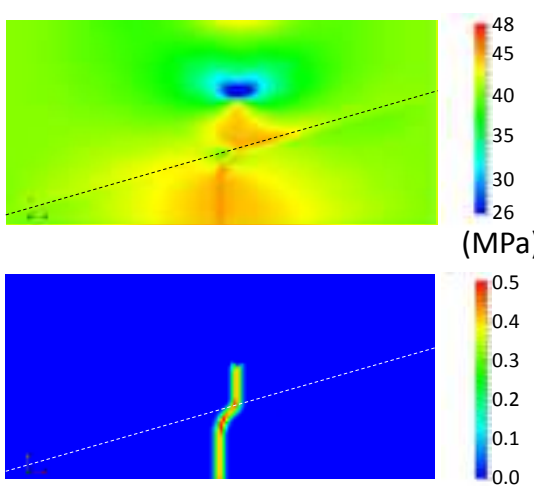

(c) after $0.5 \mathrm{sec}$ (fraction)

Fig. 9 Horizontal stress and damage distribution (layer dip angle $=15$ degree, $\mathrm{E}_{1} / \mathrm{E}_{2}=40 \mathrm{GPa} / 10 \mathrm{GPa}$, $\mathrm{K}_{\mathrm{IC} 1} / \mathrm{K}_{\mathrm{IC} 2}=1.41 \mathrm{MPa} \mathrm{m}^{0.5} / 0.5 \mathrm{MPa} \mathrm{m}^{0.5}$, principal stress difference $=1.0 \mathrm{MPa}$ ). 
Kinking angle increases with the Young's modulus contrast.

$30 \mathrm{~cm}$

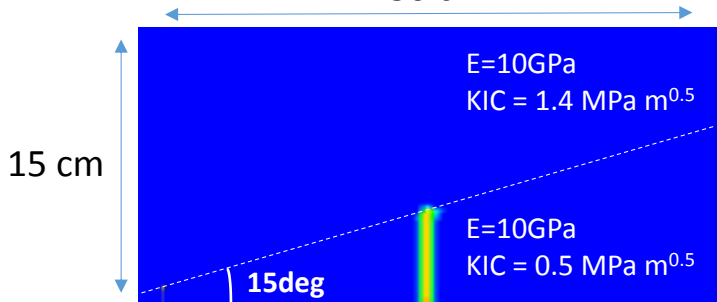

(a) $E_{1 /} E_{2}=10 G P a / 10 G P a$

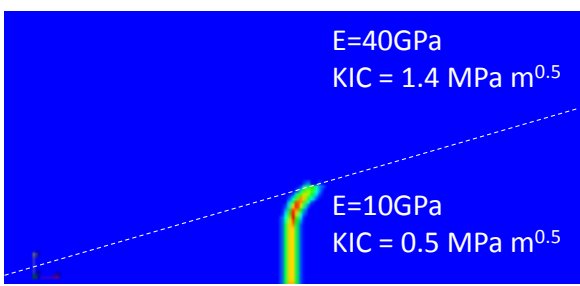

(c) $E_{1 /} E_{2}=40 G P a / 10 G P a$

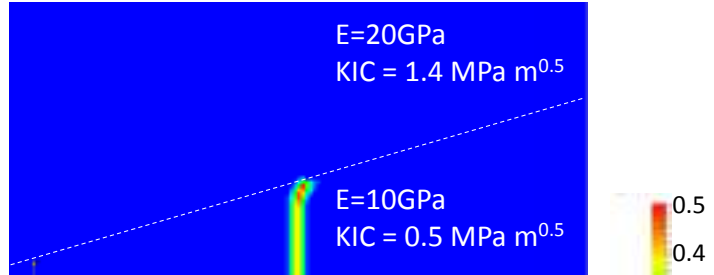

(b) $E_{1 /} E_{2}=20 G P a / 10 G P a$

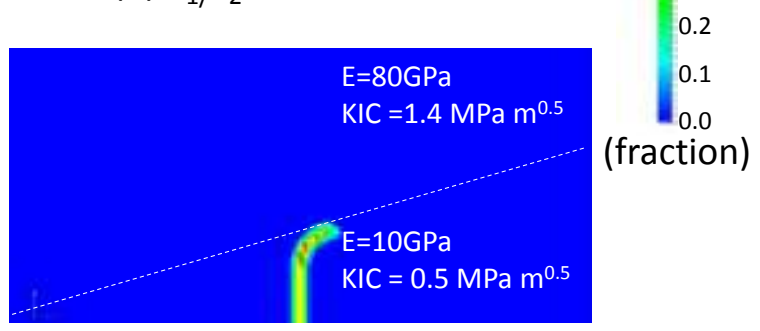

(c) $E_{1 /} E_{2}=80 \mathrm{GPa} / 10 \mathrm{GPa}$

Fig. 10 Damage distribution in the different Young's contrast cases (layer dip angle $=15$ degree, $\mathrm{K}_{\mathrm{IC} 1} / \mathrm{K}_{\mathrm{IC} 2}=1.41 \mathrm{MPa} \mathrm{m}^{0.5} / 0.5 \mathrm{MPa} \mathrm{m}{ }^{0.5}$, principal stress difference $=1.0 \mathrm{MPa}$ ).

Kinking angle does not change depending on the fracture toughness contrast.

$30 \mathrm{~cm}$

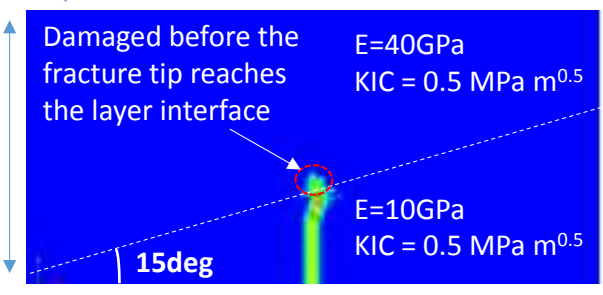

(a) $\mathrm{K}_{\mathrm{IC} 1 /} / \mathrm{K}_{\mathrm{IC2}}=0.5 \mathrm{MPa} \mathrm{m}^{0.5} / 0.5 \mathrm{MPa} \mathrm{m}^{0.5}$

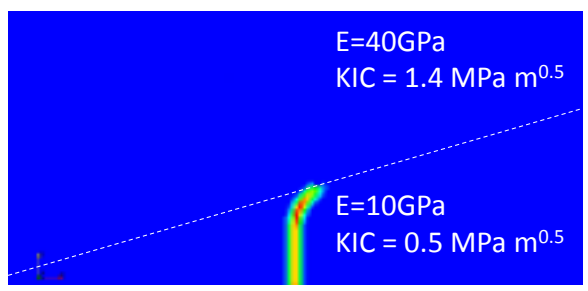

(c) $\mathrm{K}_{\mathrm{IC} 1 /} \mathrm{K}_{\mathrm{IC} 2}=1.4 \mathrm{MPa} \mathrm{m}^{0.5} / 0.5 \mathrm{MPa} \mathrm{m}^{0.5}$

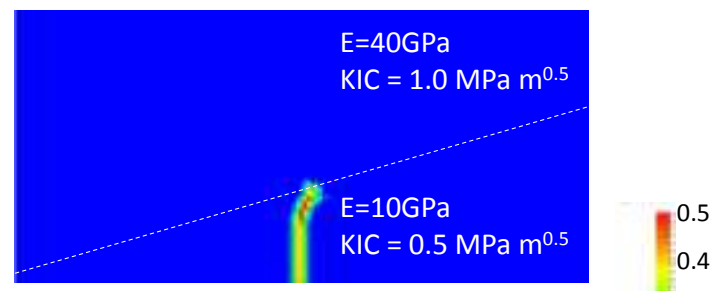

(b) $\mathrm{K}_{\mathrm{IC} 1 /} \mathrm{K}_{\mathrm{IC2}}=1.0 \mathrm{MPa} \mathrm{m}^{0.5} / 0.5 \mathrm{MPa} \mathrm{m}^{0.5} \quad 0.3$

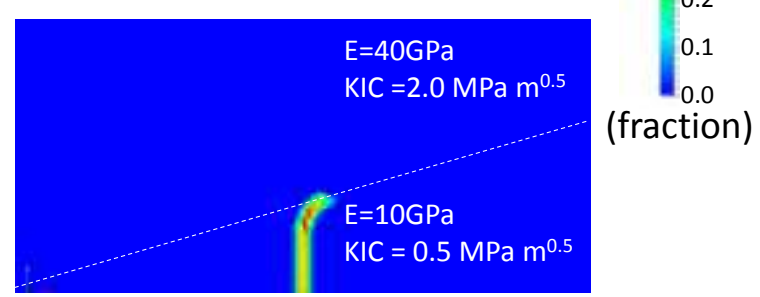

(d) $\mathrm{K}_{\mathrm{IC} 1 /} / \mathrm{K}_{\mathrm{IC2}}=2.0 \mathrm{MPa} \mathrm{m}^{0.5} / 0.5 \mathrm{MPa} \mathrm{m}^{0.5}$

Fig. 11 Damage distribution in the different fracture toughness contrast (layer dip angle $=15$ degree, $\mathrm{E}_{1} / \mathrm{E}_{2}=40 \mathrm{GPa} / 10 \mathrm{GPa}$, principal stress difference $\left.=1.0 \mathrm{MPa}\right)$. 
Kinking angle decreases with the increase of the principle stress difference.

$30 \mathrm{~cm}$

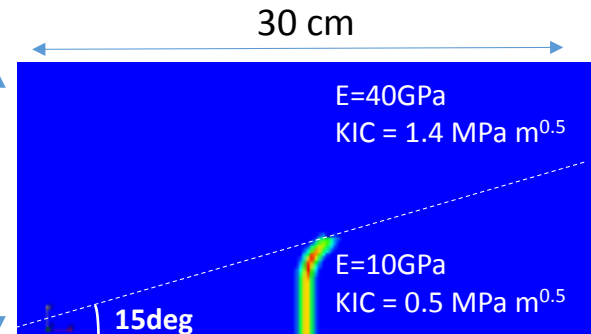

(a) principal stress difference $=1 \mathrm{MPa}$

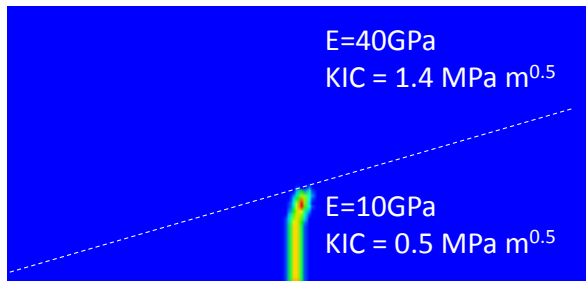

(c) principal stress difference $=10 \mathrm{MPa}$

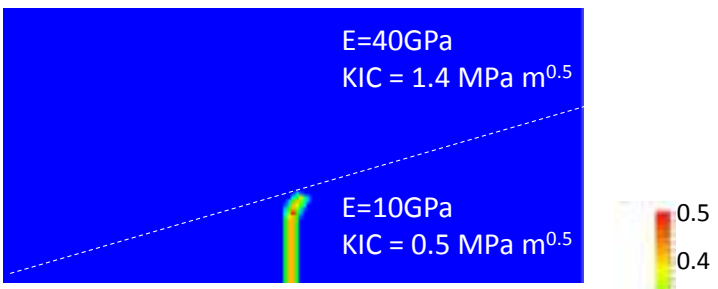

(b) principal stress difference $=6 \mathrm{MPa} \quad 0.3$

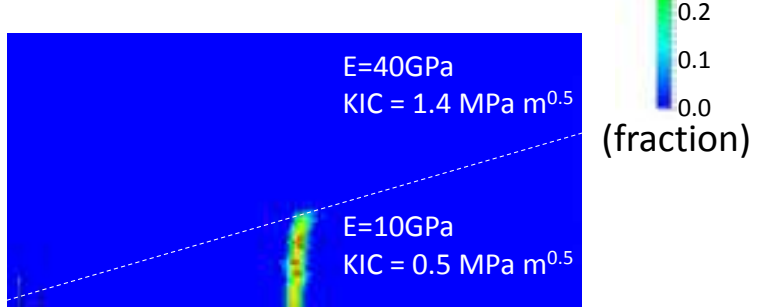

(d) principal stress difference $=20 \mathrm{MPa}$

Fig. 12 Damage distribution in the different principal stress difference cases (layer dip angle $=15$ degree, $\mathrm{E}_{1} / \mathrm{E}_{2}=40 \mathrm{GPa} / 10 \mathrm{GPa}, \mathrm{K}_{\mathrm{IC} 1} / \mathrm{K}_{\mathrm{IC} 2}=1.41 \mathrm{MPa} \mathrm{m}^{0.5} / 0.5 \mathrm{MPa}$.

Kinking angle increases with the layer dip angle.

$30 \mathrm{~cm}$

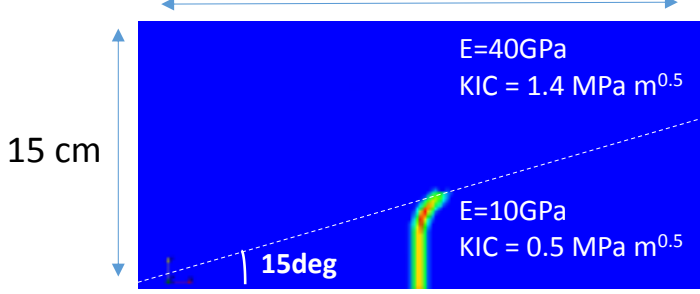

(a) $15 \mathrm{deg}$ (stress difference $=1 \mathrm{MPa}$ )

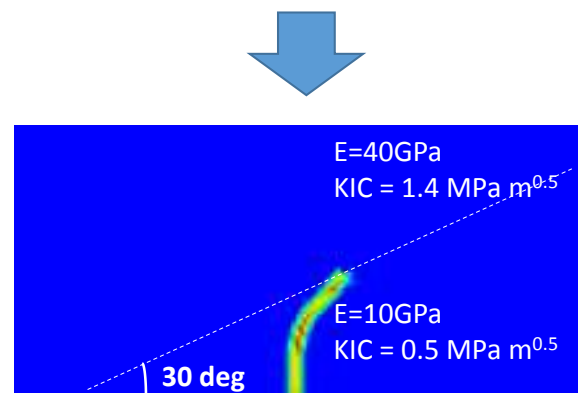

(c) 30deg (stress difference $=1 \mathrm{MPa}$ )

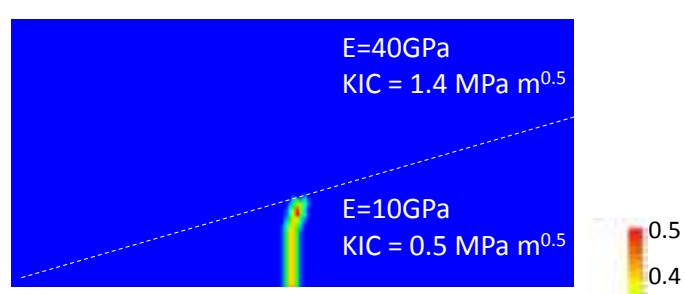

(b) $15 \mathrm{deg}$ (stress difference $=10 \mathrm{MPa} \quad 0.3$
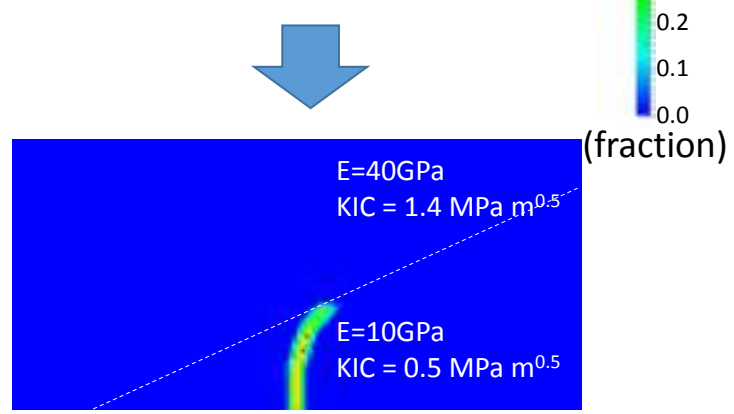

(d) $30 \mathrm{deg}$ (stress difference $=10 \mathrm{MPa}$ )

Fig. 13 Damage distribution in the different layer dip angle and different principal stress cases $\left(\mathrm{E}_{1} / \mathrm{E}_{2}=40\right.$ $\mathrm{GPa} / 10 \mathrm{GPa}, \mathrm{K}_{\mathrm{IC1}} / \mathrm{K}_{\mathrm{IC} 2}=1.41 \mathrm{MPa} \mathrm{m}^{0.5} / 0.5 \mathrm{MPa} \mathrm{m}^{0.5}$ ). 


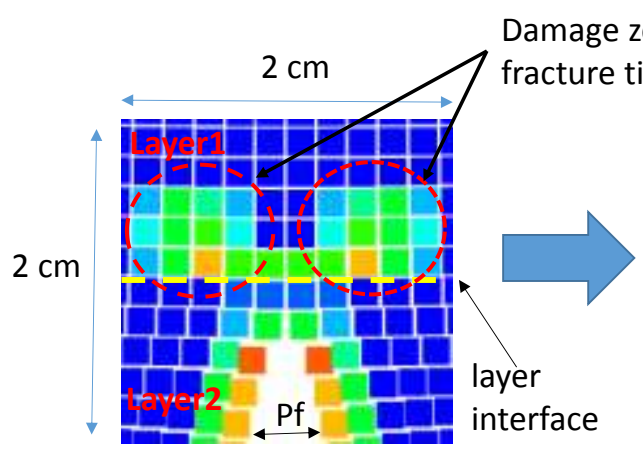

(a) time $=0.34 \mathrm{sec}$

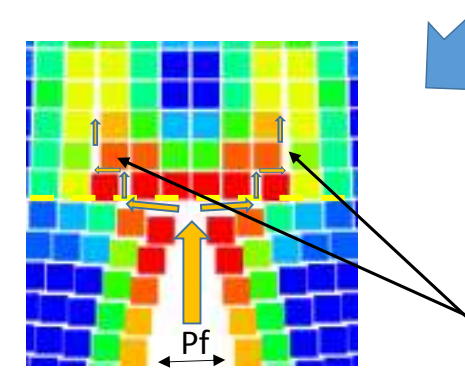

(c) time $=0.39 \mathrm{sec}$ (just after breaking)

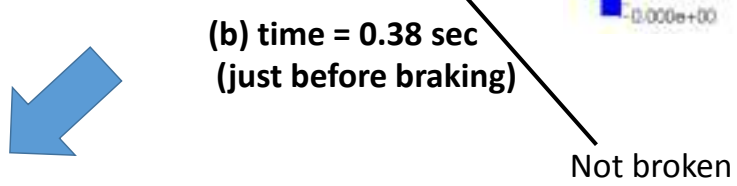

As soon as the fracture reaches the layer interface, the fracture continues to propagate into the $1^{\text {st }}$ layer following the pre-damaged zone.

Fig. 14 Damage distribution change near the fracture tip ("branching” case).

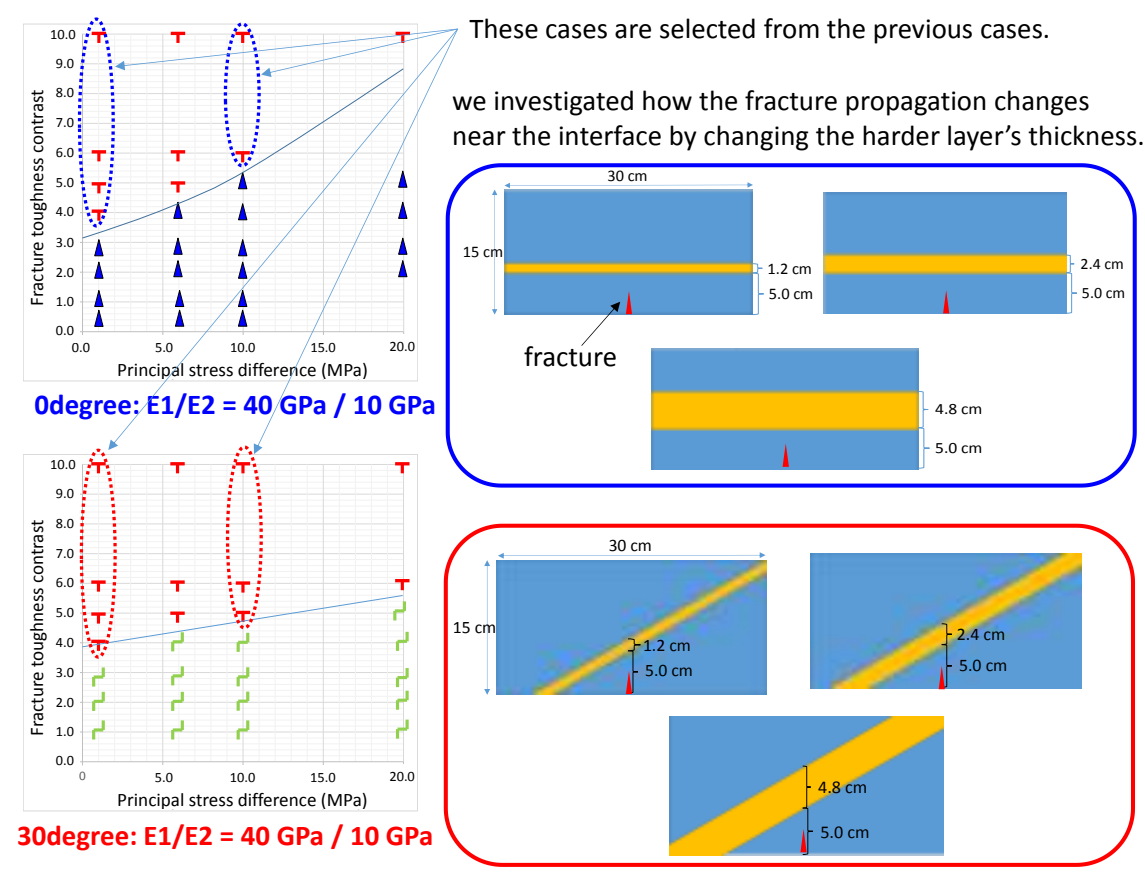

Fig. 15 Damage distribution in the different layer dip angle and different principal stress cases (E1/E2=40 $\mathrm{GPa} / 10 \mathrm{GPa}, \mathrm{KIC} 1 / \mathrm{KIC} 2=1.41 \mathrm{MPa} \mathrm{m} 0.5 / 0.5 \mathrm{MPa} \mathrm{m} 0.5)$. 


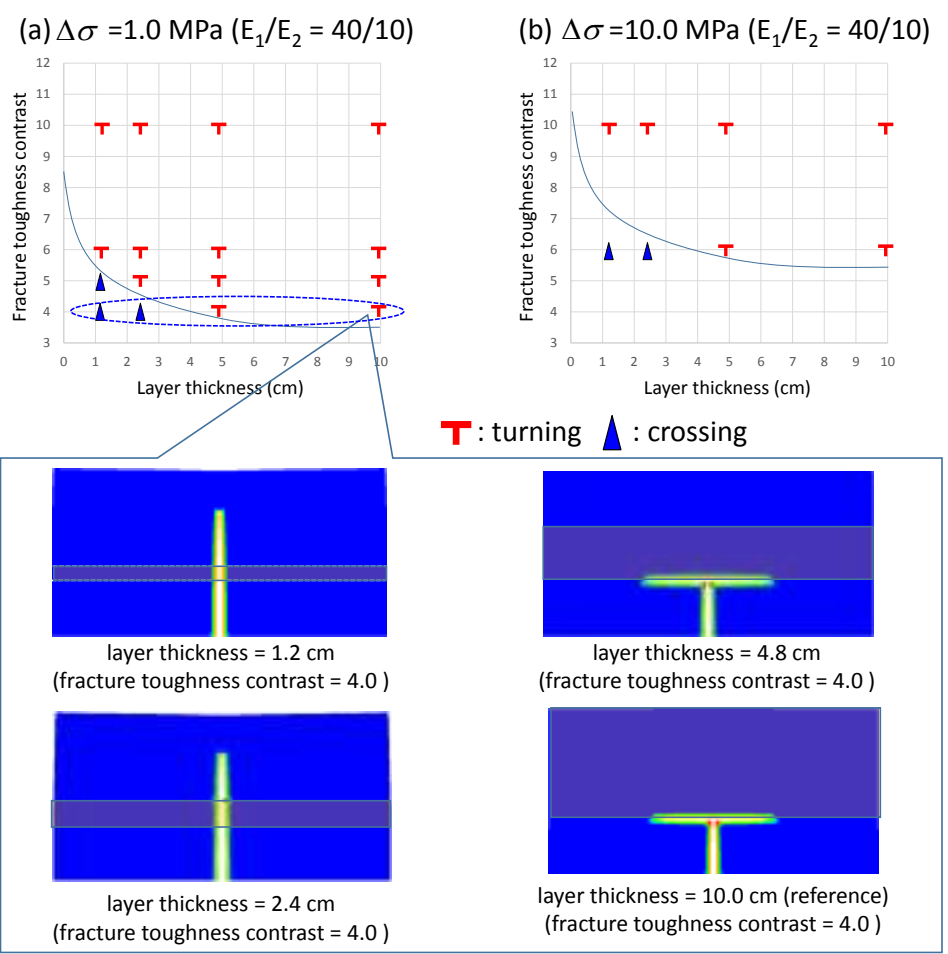

Fig. 16 Fracture propagation behavior for the different layer thickness cases ( 0 degree dip cases).

(a) $\Delta \sigma=1.0 \mathrm{MPa}\left(\mathrm{E}_{1} / \mathrm{E}_{2}=40 / 10\right)$

(b) $\Delta \sigma=10.0 \mathrm{MPa}\left(\mathrm{E}_{1} / \mathrm{E}_{2}=40 / 10\right)$

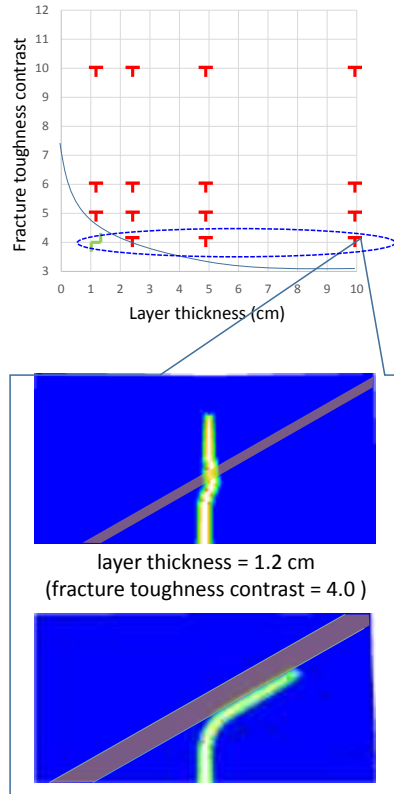

layer thickness $=2.4 \mathrm{~cm}$ (fracture toughness contrast $=4.0$ )

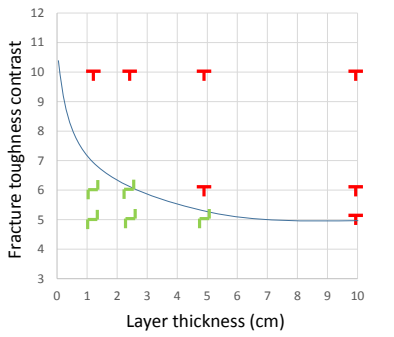

$\mathbf{T}$ : turning $\mathbf{\Lambda}$ : crossing

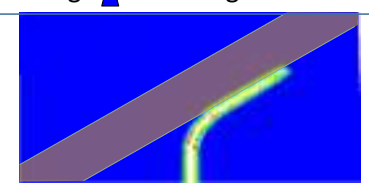

layer thickness $=4.8 \mathrm{~cm}$ $($ fracture toughness contrast $=4.0)$

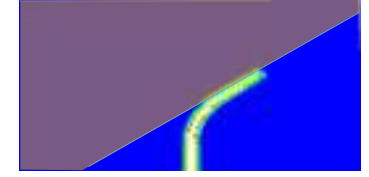

layer thickness $=10.0 \mathrm{~cm}$ (reference) (fracture toughness contrast $=4.0$ )

Fig. 17 Fracture propagation behavior for the different layer thickness cases (30 degree dip cases). 


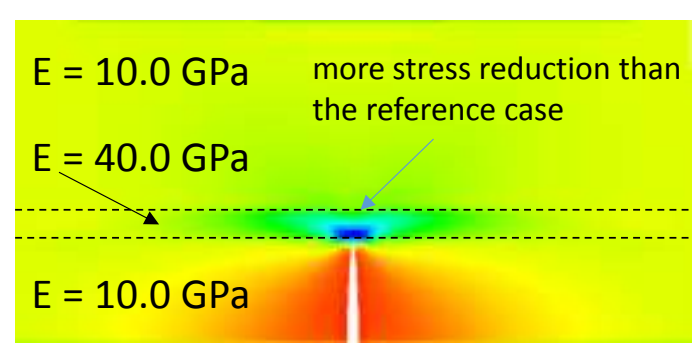

(a) layer thickness $=1.2 \mathrm{~cm}$

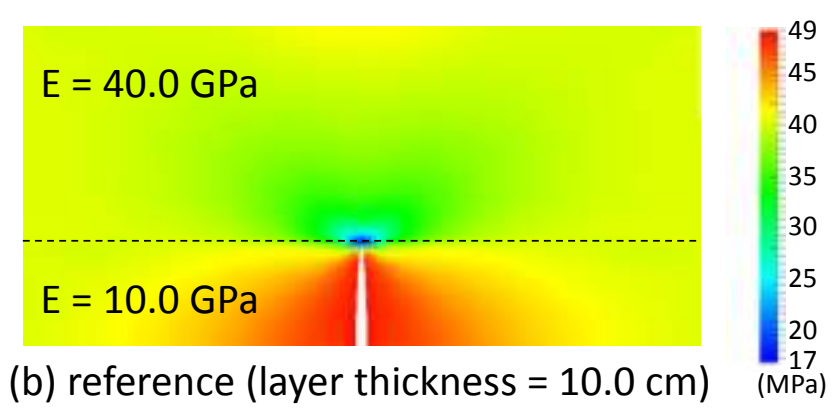

(b) reference $($ layer thickness $=10.0 \mathrm{~cm}) \quad(\mathrm{MPa})$

Fig. 18 Stress distribution near the layer interface (the thinnest layer case vs. referece case).
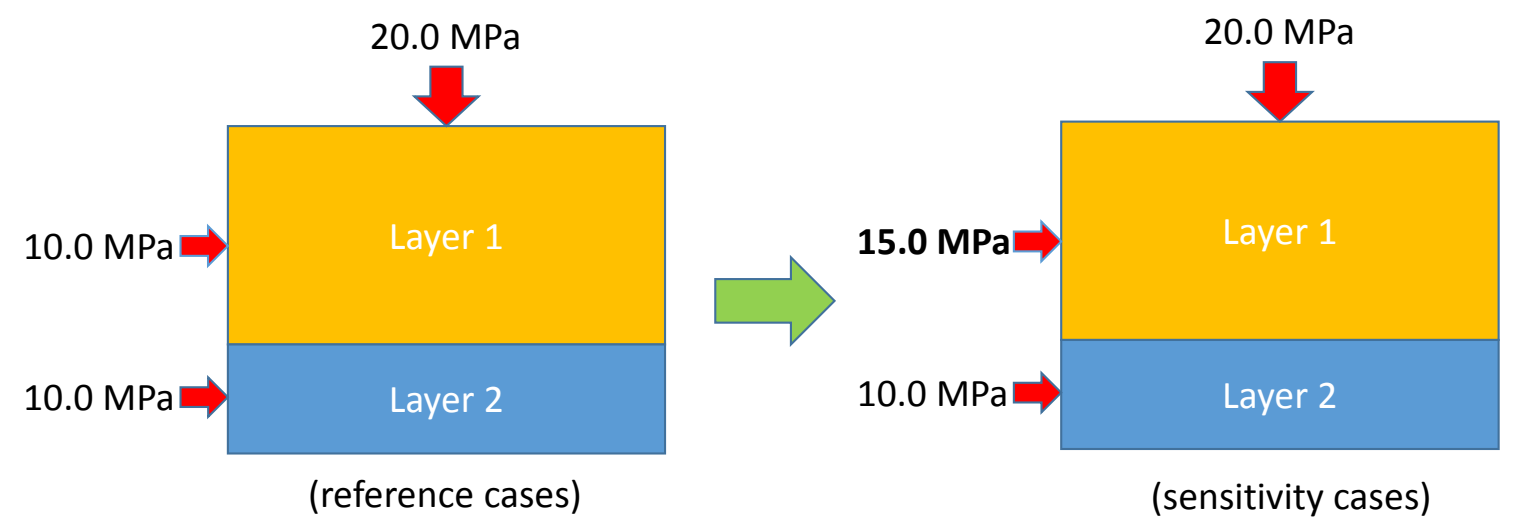

Fig. 19 Horizontal stress modification from the referece cases. 


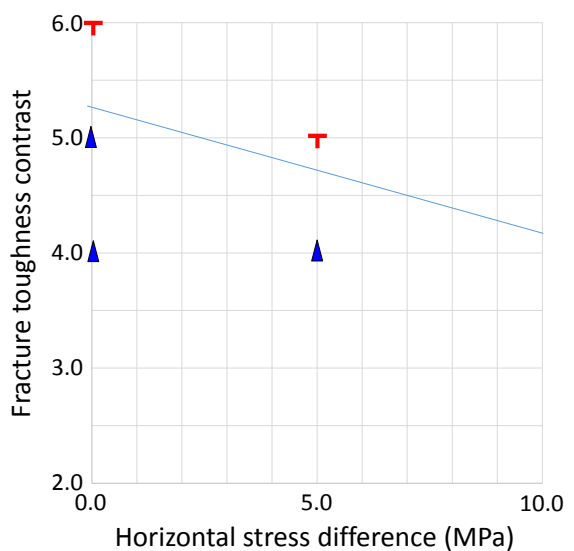

(a) $E_{1} / E_{2}=10 G P a / 10 G P a$

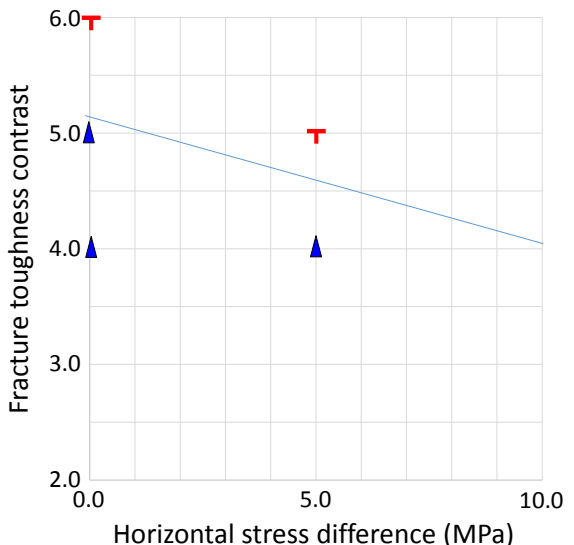

(c) $\mathrm{E}_{1} / \mathrm{E}_{2}=40 \mathrm{GPa} / 10 \mathrm{GPa}$

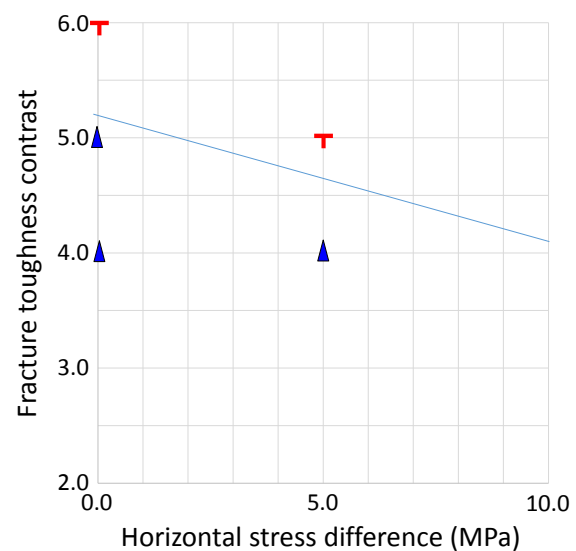

(b) $E_{1} / E_{2}=20 G P a / 10 G P a$

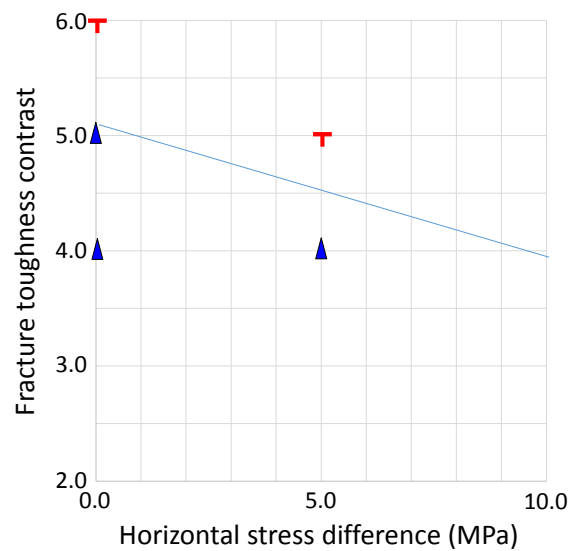

(b) $E_{1} / E_{2}=80 \mathrm{GPa} / 10 \mathrm{GPa}$

Fig. 20 Effect of horizontal stress difference. 


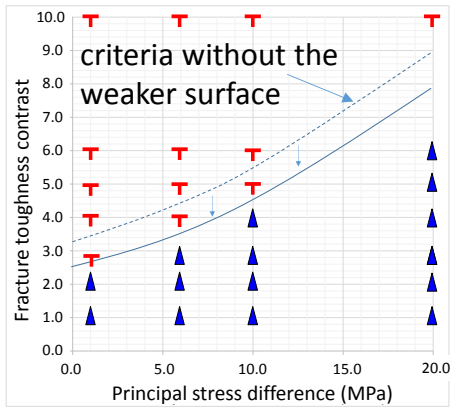

(a) $E_{1} / E_{2}=10 \mathrm{GPa} / 10 \mathrm{GPa}$

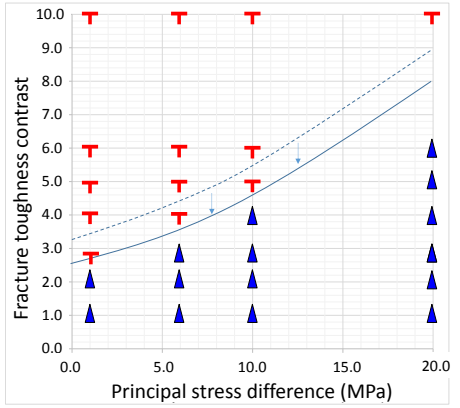

(b) $E_{1} / E_{2}=20 \mathrm{GPa} / 10 \mathrm{GPa}$

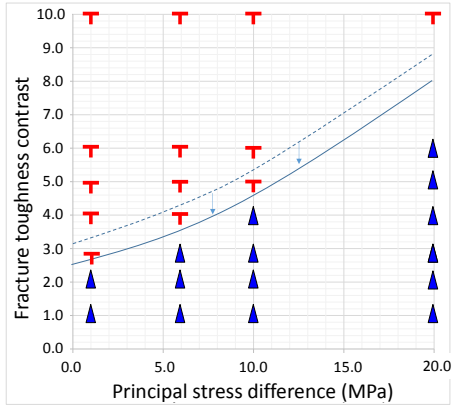

(c) $E_{1} / E_{2}=40 \mathrm{GPa} / 10 \mathrm{GPa}$

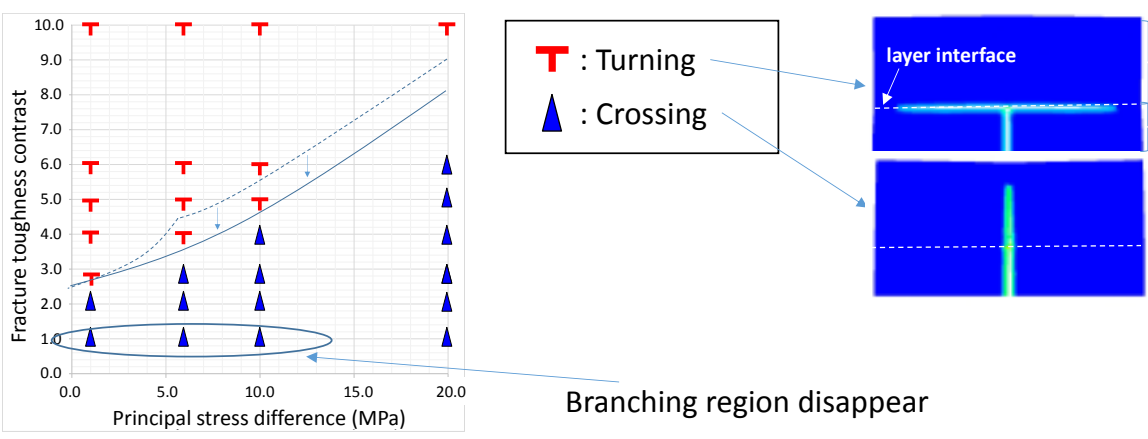

(d) $E_{1} / E_{2}=80 \mathrm{GPa} / 10 \mathrm{GPa}$

Fig. 21 Fracture turning behavior (weak surface cases: layer dip angle $=0$ degree).

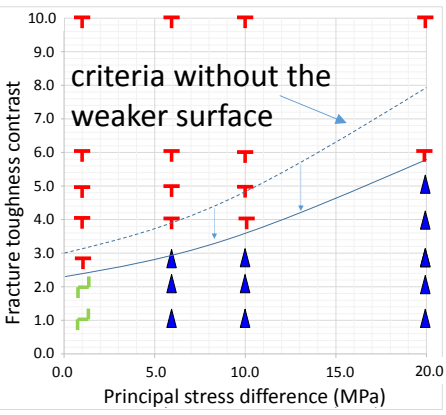

(a) $E_{1} / E_{2}=10 \mathrm{GPa} / 10 \mathrm{GPa}$

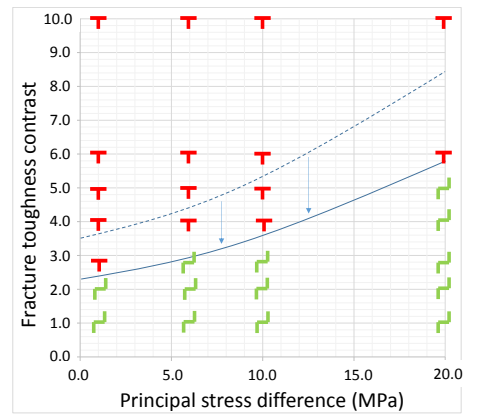

(b) $E_{1} / E_{2}=20 \mathrm{GPa} / 10 \mathrm{GPa}$

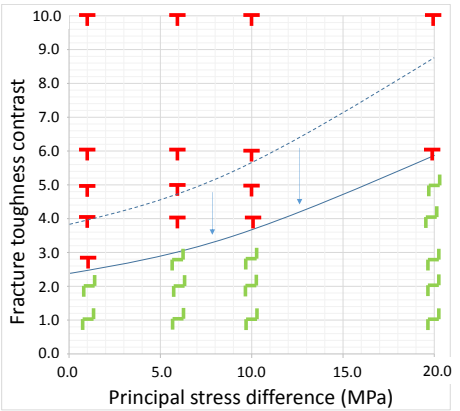

(c) $E_{1} / E_{2}=40 \mathrm{GPa} / 10 \mathrm{GPa}$

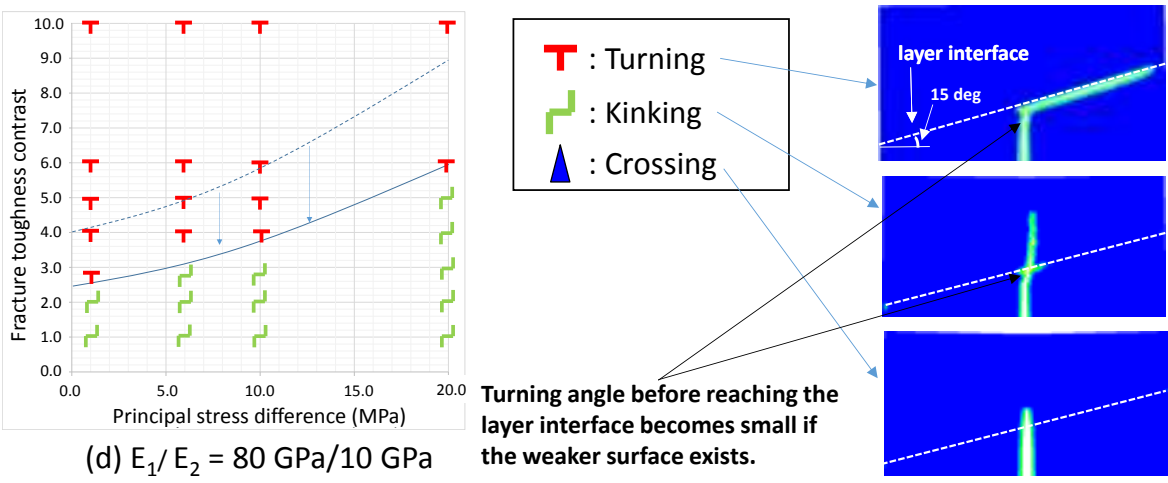

layer 1

layer 2

Fig. 22 Fracture turning behavior (weak surface cases: layer dip angle $=15$ degree) 


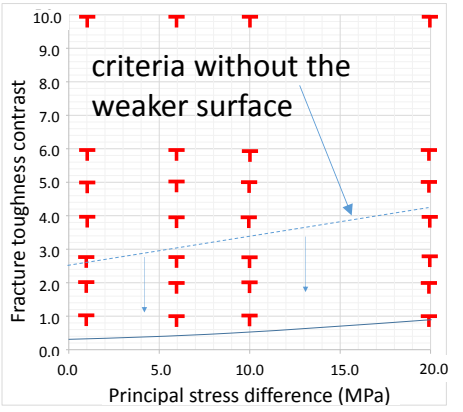

(a) $E_{1} / E_{2}=10 \mathrm{GPa} / 10 \mathrm{GPa}$

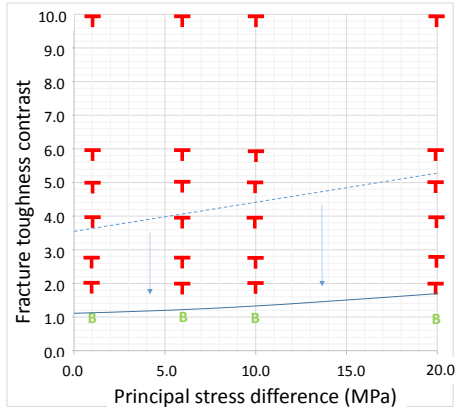

(b) $E_{1} / E_{2}=20 \mathrm{GPa} / 10 \mathrm{GPa}$

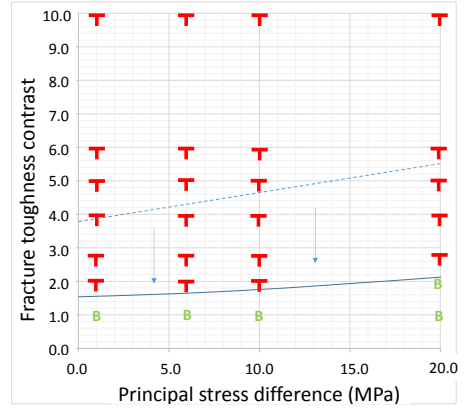

(c) $E_{1} / E_{2}=40 \mathrm{GPa} / 10 \mathrm{GPa}$
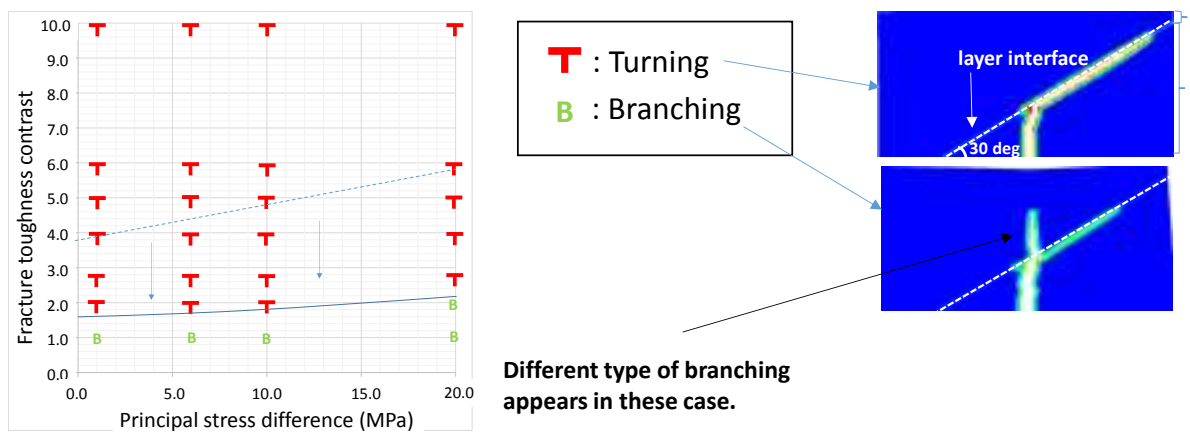

layer 1 layer 2

Different type of branching appears in these case.

(d) $E_{1} / E_{2}=80 \mathrm{GPa} / 10 \mathrm{GPa}$

Fig. 23 Fracture turning behavior (weak surface cases: layer dip angle $=30$ degree).

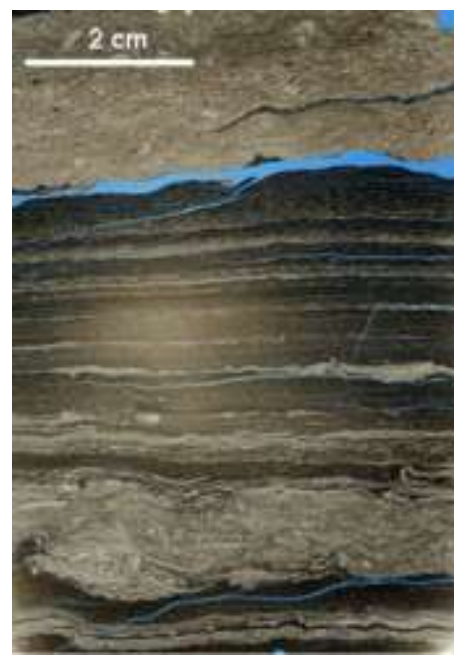

Fig. 24 Example of the $\mathrm{cm}$ order vertical heterogeneity (taken from (Passey et al., 2010)). 


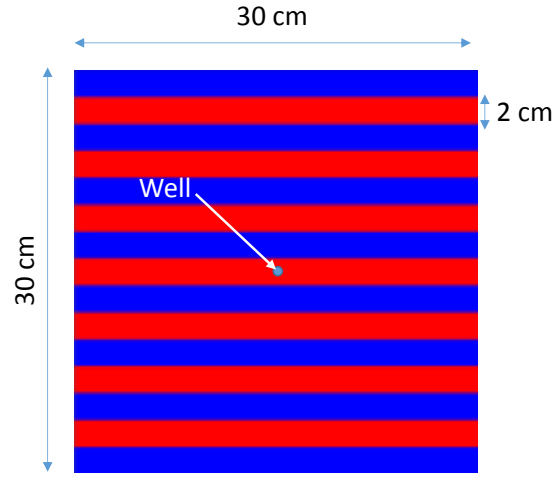

(a) 0 degree model

Higher Young's modulus

Lower Young's modulus

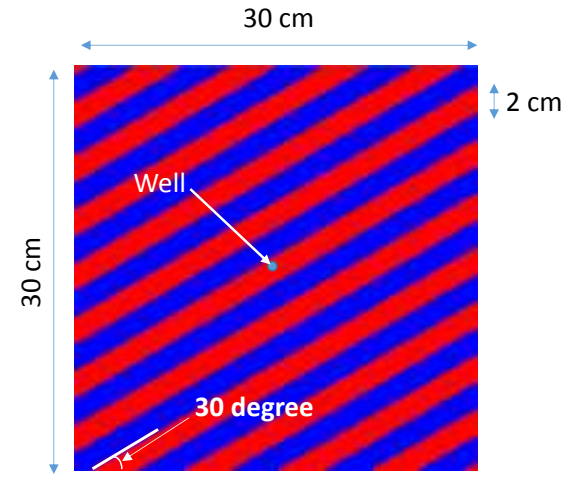

(a) 30 degree model

Two different types of mechanical properties are assigned in blue and red layers respectively.

Fig. 25 Model description (0 degree and 30 degree dip).

Initialization1: (1step initialization)

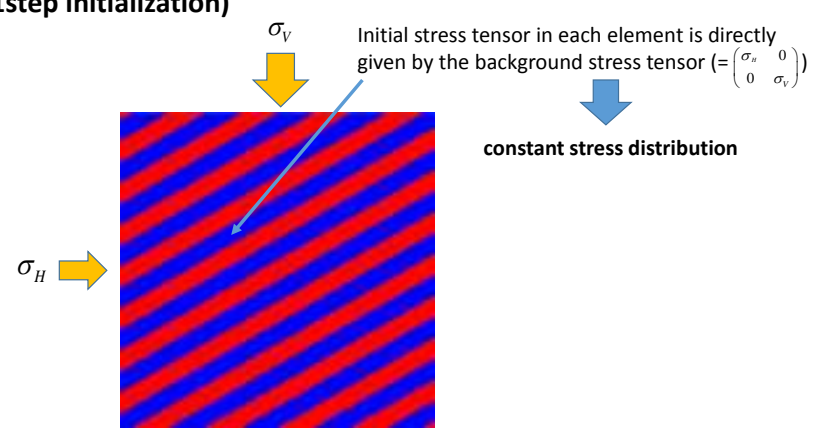

Initialization2: (2step initialization)

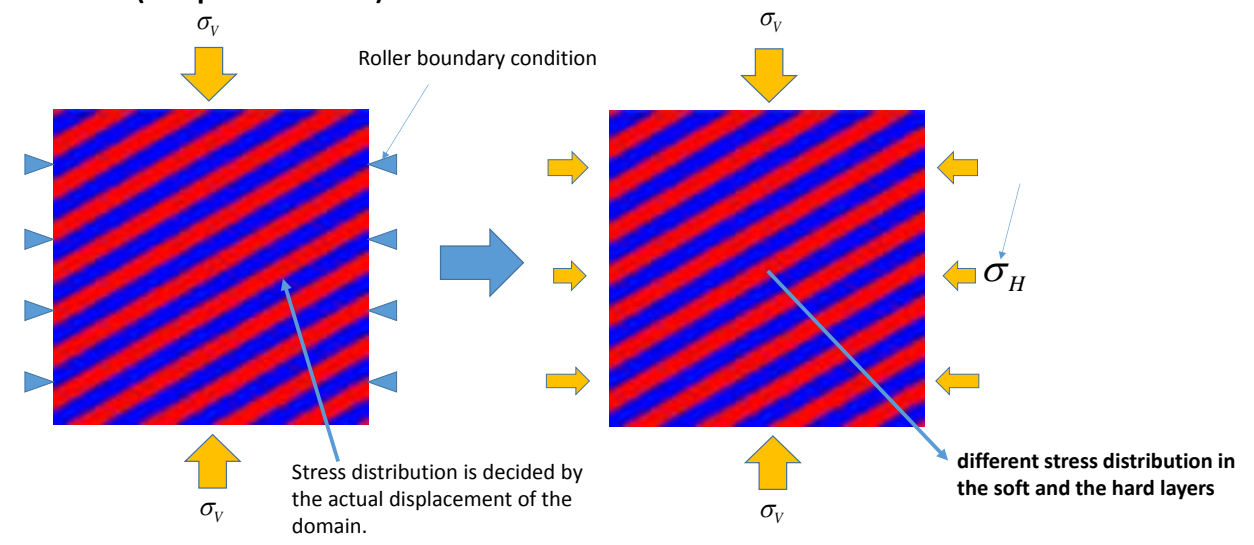

Fig. 26 Two different initialization. 


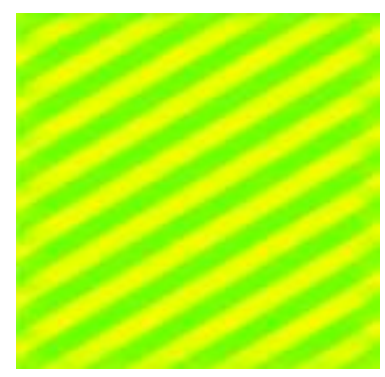

low_contrast_dipping_II $(\mathrm{E} 1=10 \mathrm{GPa}, \mathrm{E} 2=20 \mathrm{GPa})$

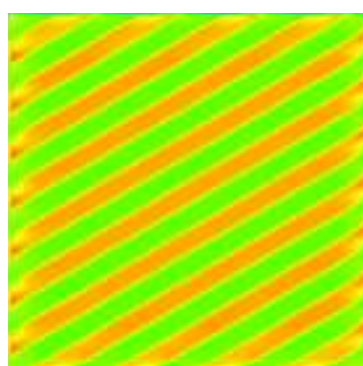

middle_contrast_dipping_II $(\mathrm{E} 1=10 \mathrm{GPa}, \mathrm{E} 2=40 \mathrm{GPa})$

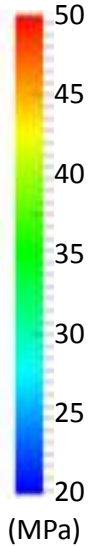

high_contrast_dipping_II \& high_contrast_2_dipping_II $(\mathrm{E} 1=10 \mathrm{GPa}, \mathrm{E} 2=80 \mathrm{GPa})$

Fig. 27 Initial horizontal stress distribution (Initialization2 Cases) 
(A) contrast_low case (after $2.0 \mathrm{sec}$ )

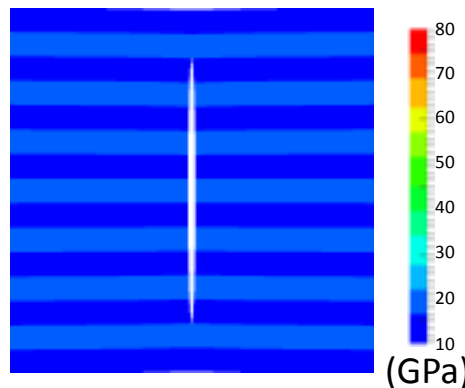

(a) Young's modulus

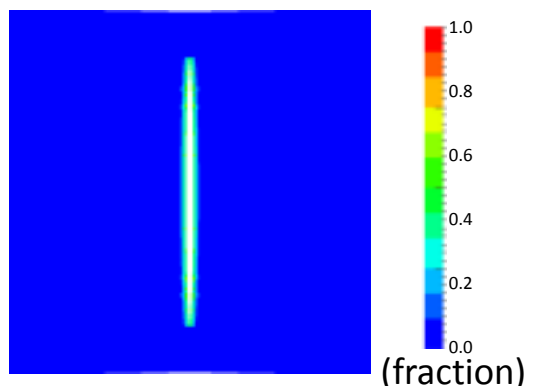

(b) Damage

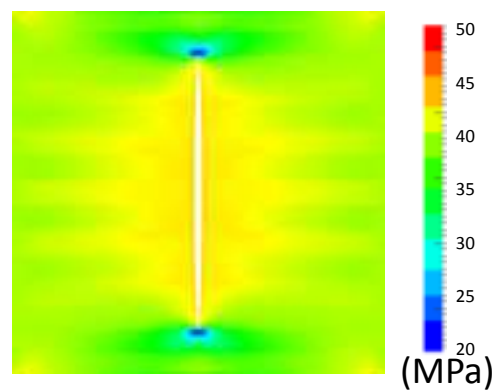

(c) $\mathrm{Sxx}(\mathrm{MPa})$

(B) contrast_middle case (after $2.0 \mathrm{sec}$ )

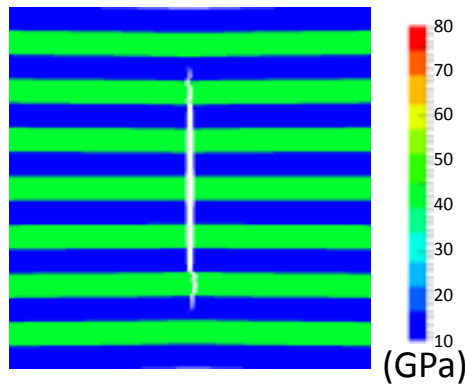

(a) Young's modulus

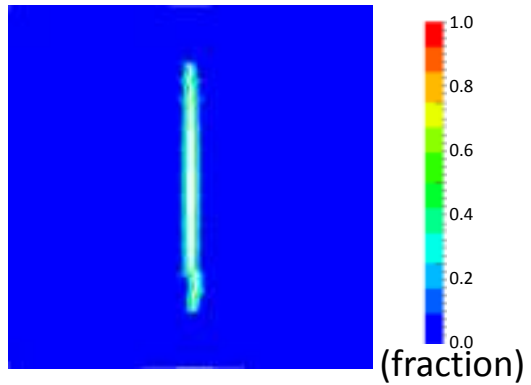

(b) Damage

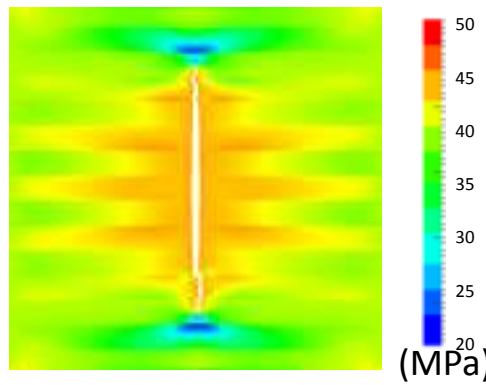

(c) Sxx (MPa)

(C) contrast_high case (after $2.0 \mathrm{sec}$ )

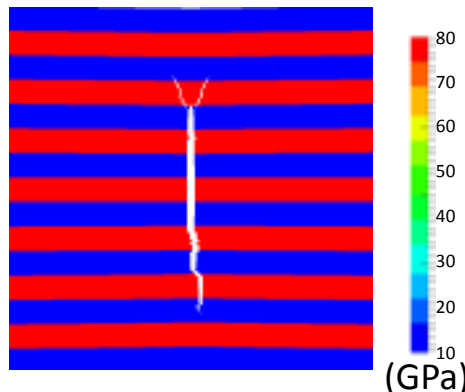

(a) Young's modulus

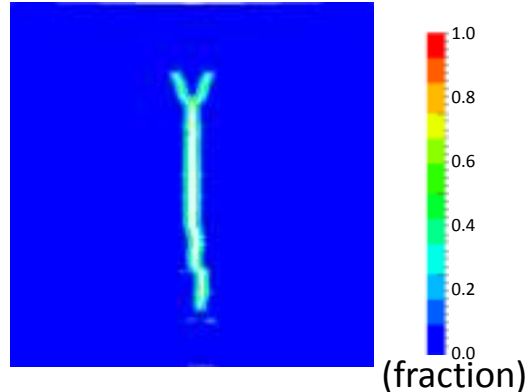

(b) Damage

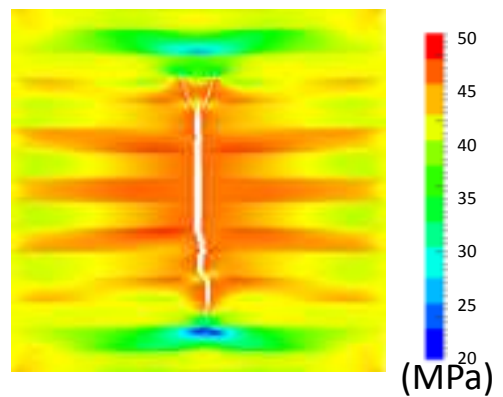

(c) Sxx (MPa)

(D) contrast_high2 case (after $1.4 \mathrm{sec}$ )

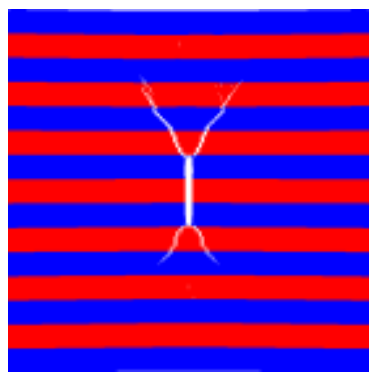

(a) Young's modulus

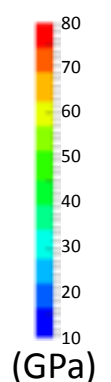

(GPa)

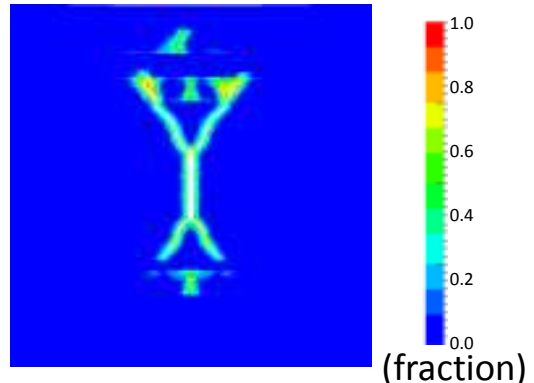

(b) Damage

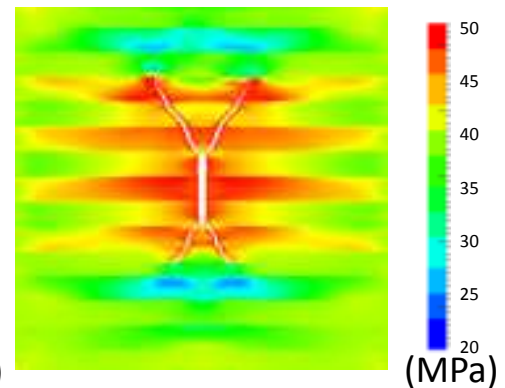

(c) $\operatorname{Sxx}(\mathrm{MPa})$

Fig. 28 Reservoir property distributions ( 0 degree cases) 
(A) contrast_low_dip case (after $2.0 \mathrm{sec}$ )

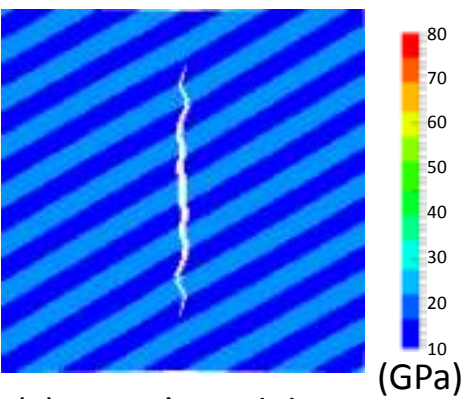

(a) Young's modulus

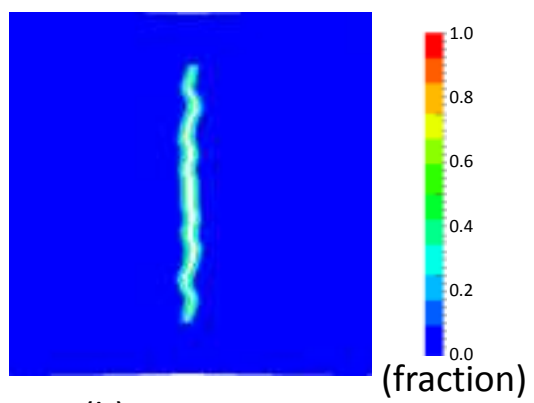

(b) Damage

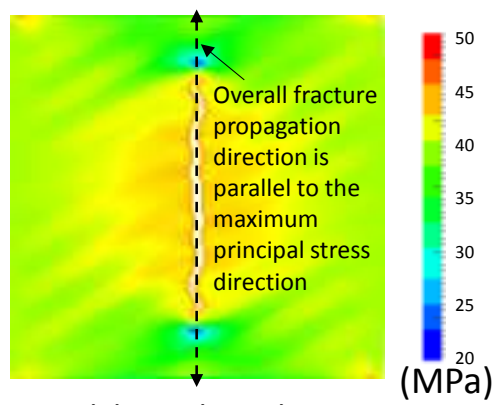

(c) $\operatorname{Sxx}(\mathrm{MPa})$

(B) contrast_middle_dip case (after $2.0 \mathrm{sec}$ )

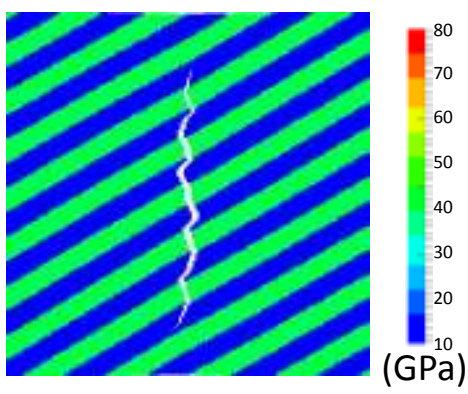

(a) Young's modulus

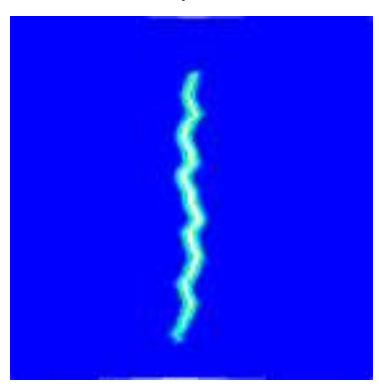

(b) Damage

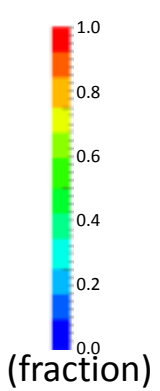

(fraction)

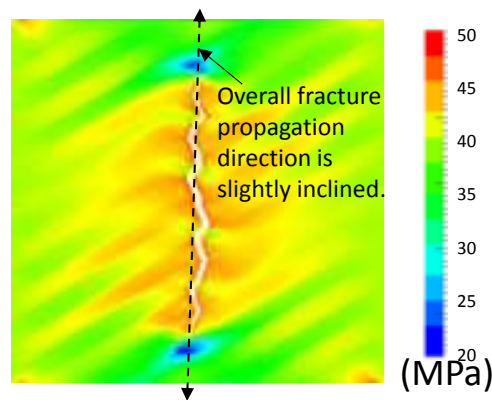

(c) $\mathrm{Sxx}(\mathrm{MPa})$

(C) contrast_high dip_case (after $2.0 \mathrm{sec}$ )

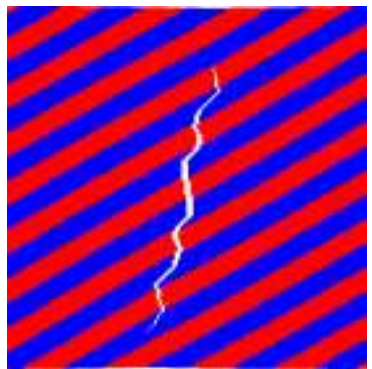

(a) Young's modulus

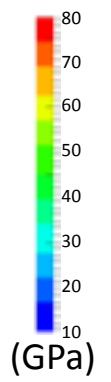

GPa)

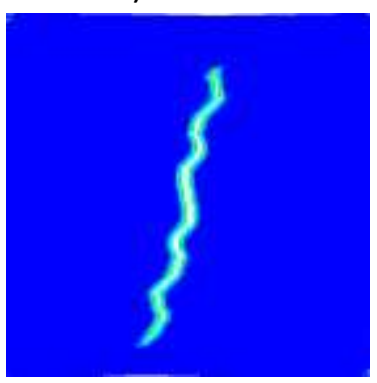

(b) Damage

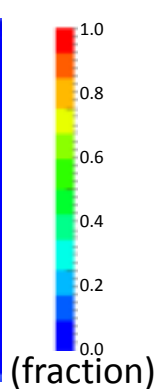

(fraction)

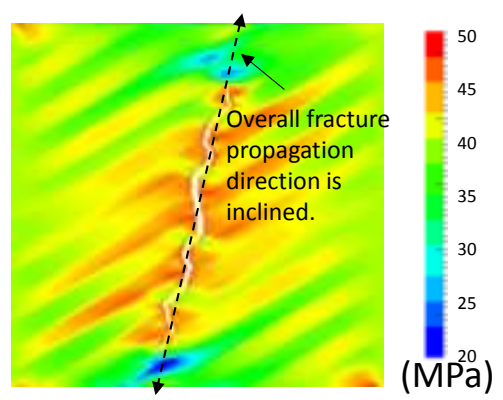

(c) Sxx (MPa)

(D) contrast_high2_dip case (after $1.0 \mathrm{sec}$ )

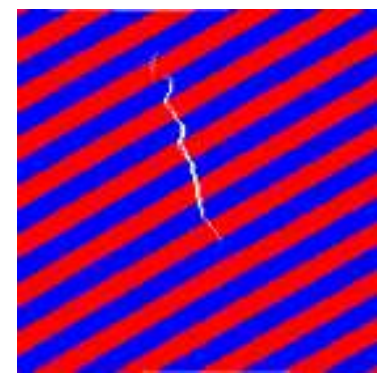

(a) Young's modulus

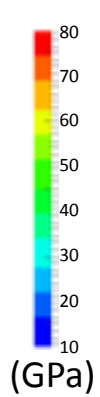

(GPa)

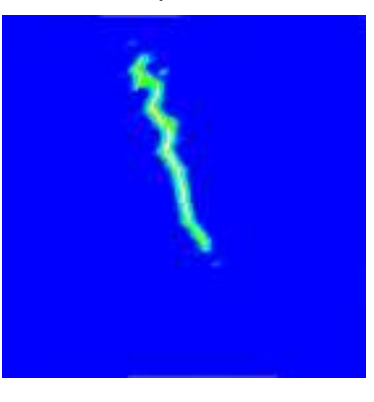

(b) Damage

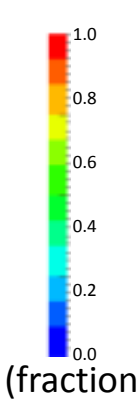

fraction

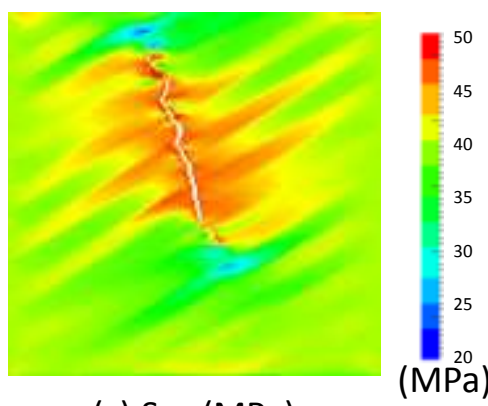

(c) Sxx (MPa)

Fig. 29 Reservoir property distributions (30 degree dip cases: Initialization Case I) 
(A) contrast_low_dip case (after $1.0 \mathrm{sec}$ )

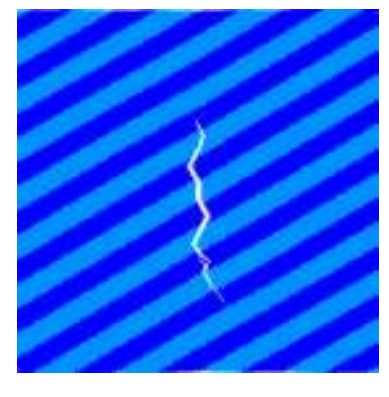

(a) Young's modulus

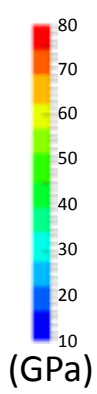

(GPa)

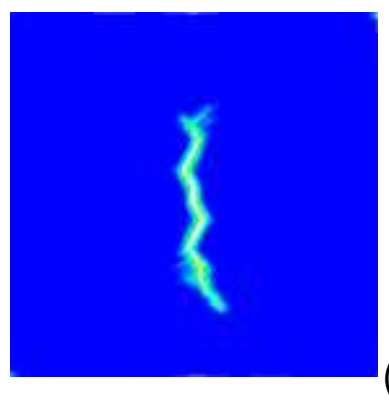

(b) Damage
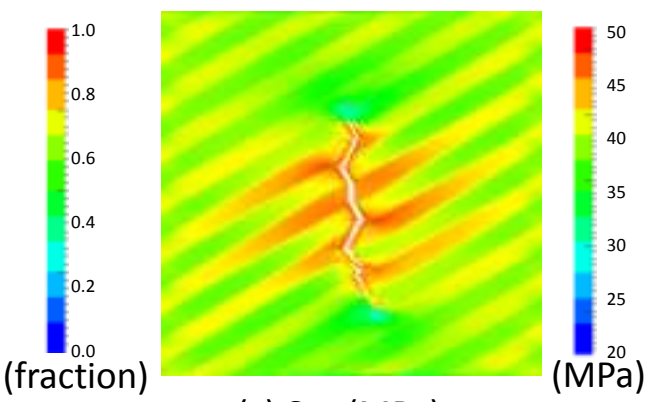

(c) $\operatorname{Sxx}(\mathrm{MPa})$

(B) contrast_middle_dip case (after $1.0 \mathrm{sec}$ )

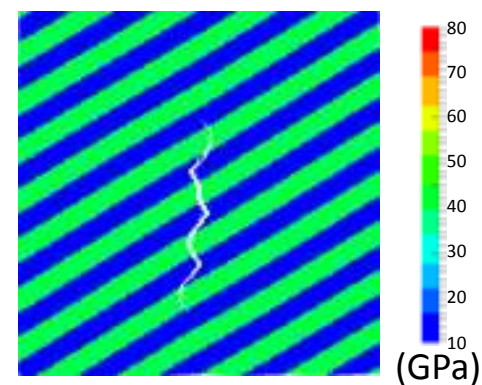

(a) Young's modulus

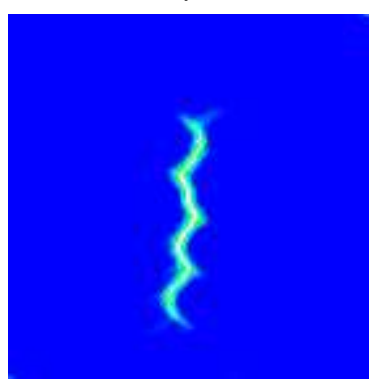

(b) Damage

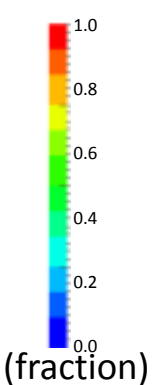

(fraction)

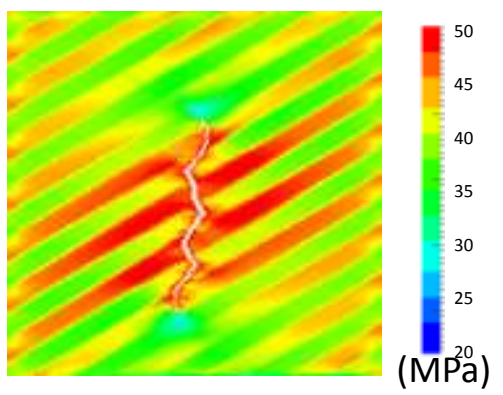

(c) $\operatorname{Sxx}(\mathrm{MPa})$

(C) contrast_high dip_case (after $1.0 \mathrm{sec}$ )

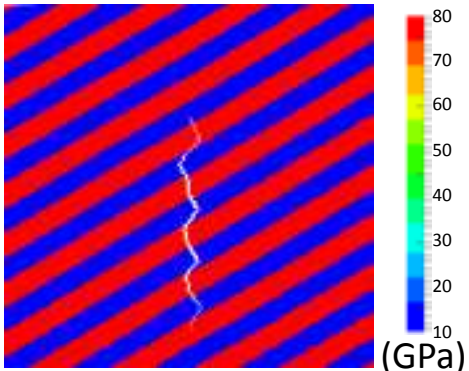

(a) Young's modulus

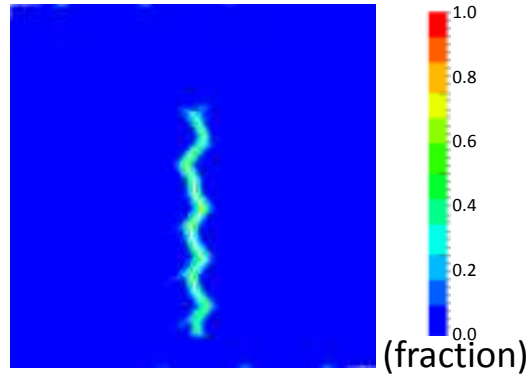

(b) Damage

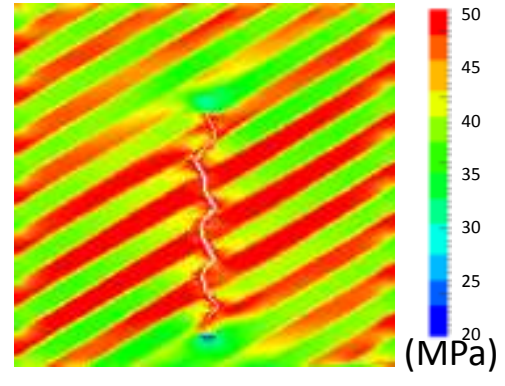

(c) $\mathrm{Sxx}(\mathrm{MPa})$

(D) contrast_high2_dip case (after $1.0 \mathrm{sec}$ )

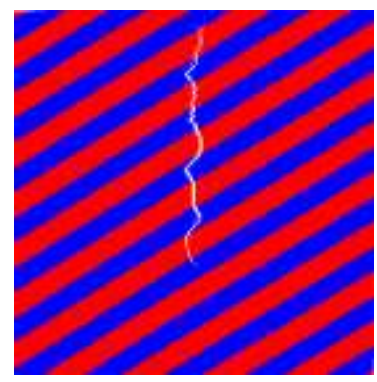

(a) Young's modulus

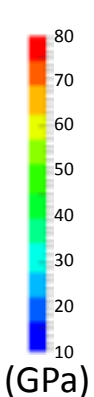

(GPa)

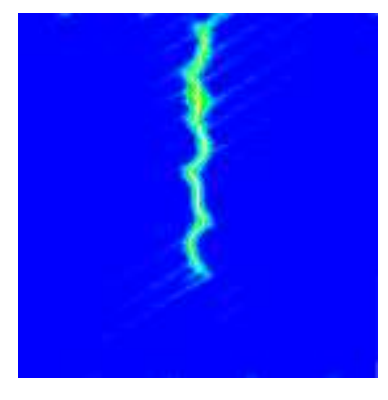

(b) Damage

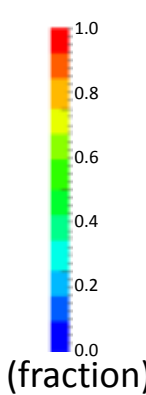

fraction)

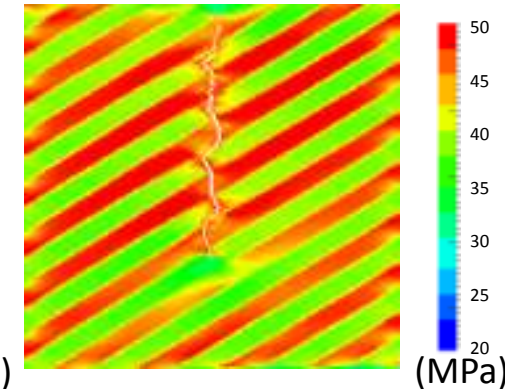

(c) $\mathrm{Sxx}(\mathrm{MPa})$

Fig. 30 Reservoir property distributions (30 degree dip cases: Initialization Case II) 


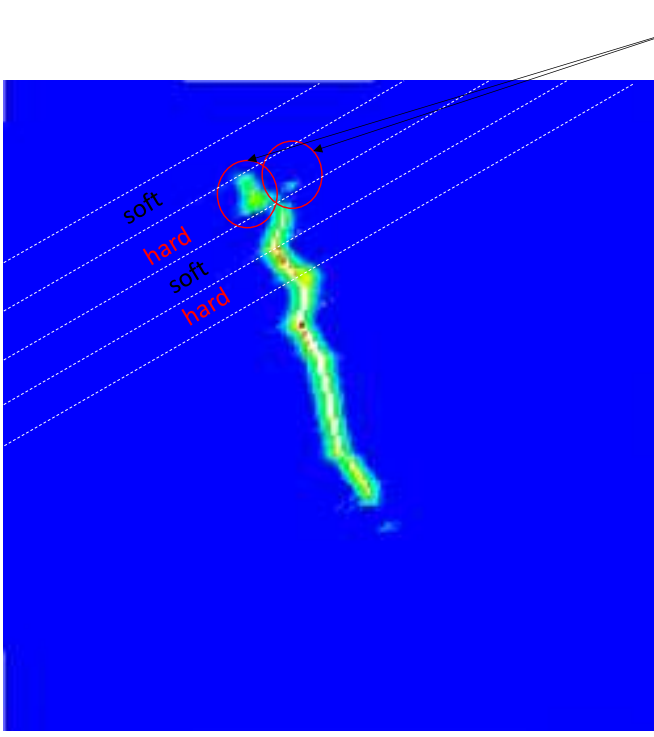

Branching like pre-damage zone appears also in the dipping layer case

However, the one side which has the closer angle to the normal angle to the softer layer grows more due to the easier deformation.

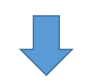

The fracture always propagates along the more pre-damaged side.

Fig. 31 Preferential fracture propagation direction (contrast_high2_dip case). 
Table 1 Parameter list for 2-layer model.

\begin{tabular}{|l|l|}
\hline Parameters & Variation \\
\hline Young's modulus in layer $1(\mathrm{GPa})$ & $10,20,40,80$ \\
\hline Young's modulus in layer $2(\mathrm{GPa})$ & $10,20,40,80$ \\
\hline Fracture toughness in layer $1\left(\mathrm{MPa} \mathrm{m}{ }^{0.5}\right)$ & $0.25,0.5,1.0,1.4,2.0,2.5,3.0,5.0$ \\
\hline Fracture toughness in layer 2 $\left(\mathrm{MPa} \mathrm{m}{ }^{0.5}\right)$ & $0.25,0.5$ \\
\hline Layer dip angle $($ degree $)$ & $0,15,30$ \\
\hline Vertical stress $(\mathrm{MPa})$ & $41,47,50,60$ \\
\hline Horizontal stress in layer 1 $(\mathrm{MPa})$ & $40,45,50$ \\
\hline
\end{tabular}

Table 2 Calculation settings.

\begin{tabular}{|c|c|}
\hline Parameter & Value \\
\hline Dimension in horizontal direction $L_{x}(\mathrm{~cm})$ & 30.0 \\
\hline Dimension in vertical direction $L_{y}(\mathrm{~cm})$ & 15.0 \\
\hline Layer 1 thickness $(\mathrm{cm})$ & 10.0 \\
\hline Layer 2 thickness $(\mathrm{cm})$ & 5.0 \\
\hline Boundary stress in vertical direction $\sigma_{V}(\mathrm{MPa})$ & Table 1 \\
\hline Boundary stress in layer 1 in horizontal direction $\sigma_{H 1} \quad(\mathrm{MPa})$ & Table 1 \\
\hline Boundary stress in layer 2 in horizontal direction $\sigma_{H_{2}}(\mathrm{MPa})$ & 40.0 \\
\hline Young's modulus in layer 1 & Table 1 \\
\hline Young's modulus in layer 2 & Table 1 \\
\hline Poisson's ratio & 0.25 \\
\hline Initial pore pressure $(\mathrm{MPa})$ & 30.0 \\
\hline Injection rate $(\mathrm{kg} / \mathrm{s})$ & 0.005 \\
\hline Fracturing fluid viscosity (cp) & 1.0 \\
\hline Medium permeability $(\mathrm{mD})$ & 0.00001 \\
\hline Number of elements & $150 \times 75$ \\
\hline Horizon size $\left(=\frac{\delta}{\Delta x}\right)$ & 3.0 \\
\hline
\end{tabular}


Table 3 List of fixed and changed parameters.

\begin{tabular}{|l|l|l|}
\hline Parameters & Condition & Variation \\
\hline Young's modulus in layer $1(\mathrm{GPa})$ & Changed & $10,20,40,80$ \\
\hline Young's modulus in layer 2 $(\mathrm{GPa})$ & Changed & $10,40,80$ \\
\hline Fracture toughness in layer $1\left(\mathrm{MPa} \mathrm{m}^{0.5}\right)$ & Changed & $0.25,0.5,1.0,1.4,2.0,2.5,3.0,5.0$ \\
\hline Fracture toughness in layer $2\left(\mathrm{MPa} \mathrm{m}^{0.5}\right)$ & Fixed & 0.5 \\
\hline Layer dip angle $($ degree $)$ & Changed & $0,15,30$ \\
\hline Vertical stress $(\mathrm{MPa})$ & Changed & $41,47,50,60$ \\
\hline Horizontal stress in layer 1 $(\mathrm{MPa})$ & Fixed & 40 \\
\hline Medium permeability $(\mathrm{mD})$ & Fixed & 0.00001 \\
\hline
\end{tabular}

Table 4 Reference case settings (investigation of the effect of layer thickness).

\begin{tabular}{|c|c|c|c|}
\hline Case Name & $\begin{array}{c}\text { Layer dip angle } \\
(\text { degree })\end{array}$ & $\begin{array}{c}\text { Principal stress } \\
\text { difference }(\mathrm{MPa})\end{array}$ & $\begin{array}{c}\text { Fracture toughness } \\
\text { contrast }\end{array}$ \\
\hline Odeg_1MPa_1 & 0.0 & 1.0 & 4.0 \\
\hline Odeg_1MPa_2 & 0.0 & 1.0 & 5.0 \\
\hline Odeg_1MPa_3 & 0.0 & 1.0 & 6.0 \\
\hline Odeg_1MPa_4 & 0.0 & 1.0 & 10.0 \\
\hline 0deg_10MPa_1 & 0.0 & 10.0 & 6.0 \\
\hline Odeg_10MPa_2 & 0.0 & 10.0 & 10.0 \\
\hline 30deg_1MPa_1 & 30.0 & 1.0 & 5.0 \\
\hline 30deg_1MPa_2 & 30.0 & 1.0 & 6.0 \\
\hline 30deg_1MPa_3 & 30.0 & 1.0 & 10.0 \\
\hline 30deg_1MPa_4 & 30.0 & 1.0 & 5.0 \\
\hline 30deg_10MPa_1 & 30.0 & 10.0 & 6.0 \\
\hline 30deg_10MPa_2 & 30.0 & 10.0 & 10.0 \\
\hline 30deg_10MPa_3 & 30.0 & 10.0 & \\
\hline
\end{tabular}


Table 5 Reference cases for the investigation of the effect of horizontal stress difference.

\begin{tabular}{|c|c|c|c|c|c|}
\hline Case Name & $\sigma_{1}(\mathrm{MPa})$ & $\sigma_{2}(\mathrm{MPa})$ & $\mathrm{E}_{1}(\mathrm{GPa})$ & $\mathrm{E}_{2}(\mathrm{GPa})$ & $\mathrm{K}_{\mathrm{IC} 1} / \mathrm{K}_{\mathrm{IC} 2}$ \\
\hline Case1_1 & 10.0 & 20.0 & 10.0 & 10.0 & 5.0 \\
\hline Case1_2 & 10.0 & 20.0 & 10.0 & 10.0 & 4.0 \\
\hline Case2_1 & 10.0 & 20.0 & 20.0 & 10.0 & 5.0 \\
\hline Case2_2 & 10.0 & 20.0 & 20.0 & 10.0 & 4.0 \\
\hline Case3_1 & 10.0 & 20.0 & 40.0 & 10.0 & 5.0 \\
\hline Case3_2 & 10.0 & 20.0 & 40.0 & 10.0 & 4.0 \\
\hline Case4_1 & 10.0 & 20.0 & 80.0 & 10.0 & 4.0 \\
\hline Case4_2 & 10.0 & 20.0 & 80.0 & 10.0 & 2.8 \\
\hline
\end{tabular}

Table 6 Case settings for multi-layer cases.

\begin{tabular}{|c|c|c|c|c|c|c|c|}
\hline Case name & $\begin{array}{c}\text { Initialization } \\
\text { method }\end{array}$ & $\begin{array}{c}\mathbf{E}_{1} \\
(\mathbf{G P a})\end{array}$ & $\begin{array}{c}\mathbf{E}_{2} \\
(\mathbf{G P a})\end{array}$ & 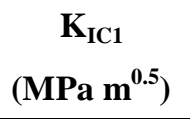 & $\begin{array}{c}\mathrm{K}_{\mathrm{IC} 2} \\
\left(\mathrm{MPa} \mathrm{\mathbf {m } ^ { 0 . 5 }}\right)\end{array}$ & $\begin{array}{c}\mathbf{G}_{\mathrm{c} 1} / \mathbf{G}_{\mathrm{c} 2} \\
(\text { hard/soft) }\end{array}$ & $\begin{array}{c}\text { Layer Dip } \\
\text { Angele ( } \theta)\end{array}$ \\
\hline low_contrast & 1 & 10 & 20 & 0.5 & 0.707 & 1.00 & 0 \\
\hline middle_contrast & 1 & 10 & 40 & 0.5 & 1.000 & 1.00 & 0 \\
\hline high_contrast & 1 & 10 & 80 & 0.5 & 1.414 & 1.00 & 0 \\
\hline high_contrast2 & 1 & 10 & 80 & 0.5 & 0.707 & 0.25 & 0 \\
\hline low_contrast_dip & 1 & 10 & 20 & 0.5 & 0.707 & 1.00 & 30 \\
\hline low_contrast_dip2 & 2 & 10 & 20 & 0.5 & 0.707 & 1.00 & 30 \\
\hline middle_contrast_dip & 1 & 10 & 40 & 0.5 & 1.000 & 1.00 & 30 \\
\hline middle_contrast_dip2 & 2 & 10 & 40 & 0.5 & 1.000 & 1.00 & 30 \\
\hline high_contras_dip & 1 & 10 & 80 & 0.5 & 1.414 & 1.00 & 30 \\
\hline high_contrast_dip2 & 2 & 10 & 80 & 0.5 & 1.414 & 1.00 & 30 \\
\hline high_contrast2_dip & 1 & 10 & 80 & 0.5 & 0.707 & 0.25 & 30 \\
\hline high_contrast2_dip2 & 2 & 10 & 80 & 0.5 & 0.707 & 0.25 & 30 \\
\hline
\end{tabular}


Table 7 Common calculation settings for multi-layer cases.

\begin{tabular}{|l|c|}
\hline \multicolumn{1}{|c|}{ Parameter } & Value \\
\hline Dimension in horizontal direction $L_{x}(\mathrm{~cm})$ & 30.0 \\
\hline Dimension in vertical direction $L_{y}(\mathrm{~cm})$ & 30.0 \\
\hline Layer thickness $(\mathrm{cm})$ & 2.0 \\
\hline Boundary stress in vertical direction $\sigma_{V}(\mathrm{MPa})$ & 60.0 \\
\hline Boundary stress in horizontal direction $\sigma_{V}(\mathrm{MPa})$ & 40.0 \\
\hline Young's modulus $E_{1}, E_{2}(\mathrm{GPa})$ & Table 6 \\
\hline Poisson's ratio & 0.25 \\
\hline Fracture toughness $K_{I C 1}, K_{I C 2}$ & Table 6 \\
\hline Initial pore pressure $P(\mathrm{MPa})$ & 28.5 \\
\hline Injection rate $(\mathrm{kg} / \mathrm{s} / \mathrm{m})$ & 0.01 \\
\hline Fracturing fluid viscosity $(\mathrm{cp})$ & 1.0 \\
\hline Medium permeability $(\mathrm{mD})$ & 0.00001 \\
\hline Number of elements & $150 \times 150$ \\
\hline Horizon size $\left(=\frac{\delta}{\Delta x}\right)$ & 3.0 \\
\hline
\end{tabular}




\section{References}

Askari, E. et al., 2008. Peridynamics for multiscale materials modeling. Journal of Physics: Conference Series, 125: 012078.

Askari, E., Xu, J. and Silling, S., 2006. Peridynamic Analysis of Damage and Failure in Composites, 44th AIAA Aerospace Sciences Meeting and Exhibit. American Institute of Aeronautics and Astronautics, Reston, Virigina, pp. 1-12.

Atkinson, B.K., 1987. Fracture mechanics of rock. Academic Press.

Bobaru, F., 2007. Influence of van der Waals forces on increasing the strength and toughness in dynamic fracture of nanofibre networks: a peridynamic approach. Modelling and Simulation in Materials Science and Engineering, 15: 397-417.

Filder, L.J., 2011. Natural Fracture Characterization of the New Albany Shale, The University of Texas at Austin. Fisher, M.K. and Warpinski, N.R., 2012. Hydraulic-Fracture-Height Growth: Real Data.

Foster, J.T., Silling, S.A. and Chen, W.N., 2011. An Energy Based Failure Criterion for Use with Peridynamic States. International Journal for Multiscale Computational Engineering, 9(6): 675-687.

Garcia, X., Nagel, N., Zhang, F., Sanchez-Nagel, M. and Lee, B., 2013. Revisiting Vertical Hydraulic Fracture Propagation Through Layered Formations - A Numerical Evaluation. American Rock Mechanics Association.

Gray Mavko, T.M., Jack Dvokin, 2009. The rock physics handbook: Tools for seismic analysis of porous media. Cambridge university press.

Gu, H. and Siebrits, E., 2008. Effect of Formation Modulus Contrast on Hydraulic Fracture Height Containment. Gunzburger, M. and Lehoucq, R., 2010. A Nonlocal Vector Calculus with Application to Nonlocal Boundary Value Problems. Multiscale Modeling \& Simulation, 8(5): 1581-1598.

Katiyar, A., Foster, J.T., Ouchi, H. and Sharma, M.M., 2014. A peridynamic formulation of pressure driven convective fluid transport in porous media. Journal of Computational Physics, 261: 209-229.

Maxwell, S.C. et al., 2009. Fault Activation During Hydraulic Fracturing. Society of Exploration Geophysicists.

Mokhtari, M., Honarpour, M.M.M., Tutuncu, A.N. and Boitnott, G.N., 2014. Acoustical and Geomechanical Characterization of Eagle Ford Shale -Anisotropy, Heterogeneity and Measurement Scale. Society of Petroleum Engineers.

Ouchi, H., 2016. Development of a Peridynamics-based Hydraulic Fracturing Model for Fracture Growth in Heterogeneous Reservoirs, The University of Texas at Austin.

Ouchi, H., Katiyar, A., Foster, J. and Sharma, M.M., 2015a. A Peridynamics model for the propagation of hydraulic fractures in heterogeneous, naturally fractured reservoirs, SPE Hydraulic Fracturing Technology Conference. Society of Petroleum Engineers.

Ouchi, H., Katiyar, A., York, J., Foster, J. and Sharma, M., 2015b. A fully coupled porous flow and geomechanics model for fluid driven cracks: a peridynamics approach. Computational Mechanics, 55(3): 561-576.

Passey, Q.R., Bohacs, K., Esch, W.L., Klimentidis, R. and Sinha, S., 2010. From Oil-Prone Source Rock to Gas-Producing Shale Reservoir - Geologic and Petrophysical Characterization of Unconventional Shale 
Gas Reservoirs. Society of Petroleum Engineers.

Rijken, P. and Cooke, M.L., 2001. Role of shale thickness on vertical connectivity of fractures: application of crack-bridging theory to the Austin Chalk, Texas. Tectonophysics, 337(1-2): 117-133.

Seleson, P.D., 2010. Multiscale Models for the Mechanics of Materials: Constitutive Relations, Upscaling from Atomistic Systems, and Interface Problems., The Florida State University.

Senseny, P.E. and Pfeifle, T.W., 1984. Fracture Toughness Of Sandstones And Shales. American Rock Mechanics Association.

Silling, S.A. and Askari, E., 2005. A meshfree method based on the peridynamic model of solid mechanics. Computers \& Structures, 83(17-18): 1526-1535.

Silling, S.a. and Bobaru, F., 2005. Peridynamic modeling of membranes and fibers. International Journal of Non-Linear Mechanics, 40: 395-409.

Silling, S.A., Epton, M., Weckner, O., Xu, J. and Askari, E., 2007. Peridynamic States and Constitutive Modeling. Journal of Elasticity, 88(2): 151-184.

Silling, S.a., Weckner, O., Askari, E. and Bobaru, F., 2010a. Crack nucleation in a peridynamic solid. International Journal of Fracture, 162: 219-227.

Silling, S.A., Weckner, O., Askari, E. and Bobaru, F., 2010b. Crack nucleation in a peridynamic solid. International Journal of Fracture, 162(1-2): 219-227.

Thiercelin, M., Jeffrey, R.G. and Naceur, K.B., 1989. Influence of Fracture Toughness on the Geometry of Hydraulic Fractures.

Tran, D., Settari, A. and Nghiem, L., 2004. New Iterative Coupling Between a Reservoir Simulator and a Geomechanics Module. SPE Journal, 9: 362-369.

Turner, D.Z., 2012. Modeling Reactive Transport in Deformable Porous Media Using the Theory of Interacting Continua. 1-38.

van Eekelen, H.A.M., 1982. Hydraulic Fracture Geometry: Fracture Containment in Layered Formations.

Walton, I. and McLennan, J., 2013. The Role of Natural Fractures in Shale Gas Production. International Society for Rock Mechanics.

Wu, H., Chudnovsky, A., Dudley, J.W. and Wong, G.K., 2004. A Map Of Fracture Behavior In The Vicinity Of An Interface. American Rock Mechanics Association.

Xu, J., Askari, A., Weckner, O., Razi, H. and Silling, S., 2007. Damage and Failure Analysis of Composite Laminates under Biaxial Loads, 48th AIAA/ASME/ASCE/AHS/ASC Structures, Structural Dynamics, and Materials Conference. American Institute of Aeronautics and Astronautics, Reston, Virigina, pp. $1-9$.

Zhang, X., Jeffrey, R.G. and Thiercelin, M., 2007. Deflection and propagation of fluid-driven fractures at frictional bedding interfaces: A numerical investigation. Journal of Structural Geology, 29(3): 396-410. 